\title{
Score equivalence of paper-, tablet-, and interactive voice response system-based versions of PROMIS, PRO-CTCAE, and numerical rating scales among cancer patients
}

\author{
Minji K. Lee ${ }^{1 *} \mathbb{C}$, Timothy J. Beebe ${ }^{2}$, Kathleen J. Yost ${ }^{1}$, David T. Eton ${ }^{1}$, Paul J. Novotny ${ }^{1}$, Amylou C. Dueck ${ }^{3}$,
} Marlene Frost ${ }^{1}$ and Jeff A. Sloan ${ }^{1}$

\begin{abstract}
Background: The study tests the effects of data collection modes on patient responses associated with the multiitem measures such as Patient-Reported Outcomes Measurement System (PROMIS ${ }^{\circledR}$ ), and single-item measures such as Patient-Reported Outcomes version of the Common Terminology Criteria for Adverse Events (PRO-CTCAE), and Numerical Rating Scale (NRS) measures.

Methods: Adult cancer patients were recruited from five cancer centers and administered measures of anxiety, depression, fatigue, sleep disturbance, pain intensity, pain interference, ability to participate in social roles and activities, global mental and physical health, and physical function. Patients were randomized to complete the measures on paper (595), interactive voice response (IVR, 596) system, or tablet computer (589). We evaluated differential item functioning (DIF) by method of data collection using the R software package, lordif. For constructs that showed no DIF, we concluded equivalence across modes if the equivalence margin, defined as $\pm 0.20 \times$ pooled SD, completely surrounds $95 \%$ confidence intervals (Cl's) for difference in mean score. If the $95 \% \mathrm{Cl}$ fell totally outside the equivalence margin, we concluded systematic score difference by modes. If the $95 \% \mathrm{Cl}$ partly overlaps the equivalence margin, we concluded neither equivalence nor difference.

Results: For all constructs, no DIF of any kind was found for the three modes. The scores on paper and tablet were more comparable than between IVR and other modes but none of the $95 \%$ Cl's were completely outside the equivalence margins, in which we established neither equivalence nor difference. Percentages of missing values were comparable for paper and tablet modes. Percentages of missing values were higher for IVR ( $2.3 \%$ to $6.5 \%$ depending on measures) compared to paper and tablet modes ( $0.7 \%$ to $3.3 \%$ depending on measures and modes), which was attributed to random technical difficulties experienced in some centers.

Conclusion: Across all mode comparisons, there were some measures with Cl's not completely contained within the margin of small effect. Two visual modes agreed more than visual-auditory pairs. IVR may induce differences in scores unrelated to constructs being measured in comparison with paper and tablet. The users of the surveys should consider using IVR only when paper and computer administration is not feasible.
\end{abstract}

\footnotetext{
*Correspondence: lee.minji@mayo.edu

${ }^{1}$ Department of Quantitative Health Sciences, Mayo Clinic, 200 First St

SW, Rochester, MN 55905, USA

Full list of author information is available at the end of the article
} 
Keywords: Mode effect, Mode of administration, Patient-reported outcomes, PRO-CTCAE, PROMIS, Numerical rating scale, Paper, Interactive voice response, Tablet computer, Differential item functioning

\section{Background}

Capturing patients' perspectives of quality of life (QOL) effectively and efficiently is critical to designing and evaluating interventions to ameliorate the impact of cancer and its treatments. Patient-reported outcomes (PROs) provide a unique method of collecting these patient perspectives directly from the patient and without interpretation by health care providers or others. One of the issues being addressed in the PRO literature is whether the assumption that items are related to the construct in identical ways for all individuals when an instrument originally developed and used for a certain mode is modified for other modes of administration. A common method is paper and pencil self-administered questionnaire (PSAQ), in which the respondent marks responses on a paper questionnaire. Computerized self-administered questionnaire (CSAQ) is a method of data collection in which the respondent uses a computer (or mobile device) to complete a questionnaire. Interactive voice recording (IVR) system, an automated telephone system navigates the respondent through the questionnaire with recording of the questions and response options,an alternative to computer-based data collection that allows a computer to detect voice and/or keypad inputs via telephone-brings about a myriad of other potential virtues such as convenience, affordability, reliability, and clinically feasibility. There have been recommendations to administer PROs electronically when possible in adult oncology [1], because it enables a comprehensive process for screening, feedback system with scores available to patients and/or providers in a timely fashion, service provision, and data management [1-3].

Recent studies, systematic reviews, and meta-analyses evaluating the equivalence of paper- versus computerbased electronic administration of PRO measures have found evidence of equivalence between the two [4-10]. However, most of these authors indicated that their findings could not be generalized to all forms of electronic PRO administration and all called for further testing of how PROs vary across data collection modes using randomized comparability trials.

Some studies evaluated the equivalence of the visual formats associated with paper- and computer screenbased administration and aural formats such as IVR [1113]: With 112 patients answering 28 Patient-Reported Outcomes version of the Common Terminology Criteria for Adverse Events (PRO-CTCAE) items in three formats (i.e., paper-, computer screen-, and IVR administration), Bennett, Dueck, Mitchell et al. [11] showed moderate to high equivalence among modes using randomized crossover design, in which each participant answered the same questionnaires with more than one mode. One limitation of their study is that the screen-based or IVR questionnaires incorporated conditional branching or skip patterns that paper mode did not, which may induce mode-specific response style or non-response. Lundy, Coons, Flood et al. [13] concluded mode equivalence for paper, handheld, tablet, IVR, and web for EQ-5D-5L, in which each participant answered the questionnaire using three modes. The order of the modes was varied among participants. However, there is possibility that participants recalled their responses to the previous set of questions answered with different modes. Bjorner, Rose, Gandek, et al. [12] used a randomized crossover design where 923 adults answered parallel Patient reported Outcomes Measurement Information System (PROMIS ${ }^{\circledR}$ ) static forms in fatigue, depression, and physical function using IVR, paper, personal digital assistant, or personal computer. They supported lack of differential item functioning in three PROMIS domains using multigroup confirmatory factor analysis and item response theory (IRT) as well as lack of clinically significant score differences across modes.

In the clinical realm, IVR has a host of features such as ease of access, increased perceived anonymity and privacy, and greater researcher control than other modes [14]. The principal downside to the use of IVR in clinical settings is that researchers cannot assume that an instrument that has been shown to have intended dimensionality, reliability, responsiveness, or interpretability using visual format has same qualities in IVRS [14]. Weiler et al. [15] found that, while there were no differences in the amount of symptoms recorded by IVR versus paper versions of allergic rhinitis response diaries, there were more missing data with the IVR, and patients overwhelmingly preferred to enter their data via paper-and-pencil. In comparing three versions of the CAHPS survey (standard print, illustration-enhanced, and telephone IVR), Shea et al. [16] found that administration times were shorter for IVR among individuals with low literacy levels. However, longer administration times were seen for IVR relative to its paper counterparts for Spanish speakers with 
high literacy levels, while the completion times were similar across modes for English speakers with high literacy levels.

One must be mindful of the possible effects of switching administration modes and plan to formally evaluate the effects of switching modes on non-response (both scale-level and item-level) and measurement error. To our knowledge, there have not been studies investigating the mode effects for numerical rating scales (NRS). In addition, the current study systematically tests the effect of three data collection methods (i.e., PSAQ, CSAQ which is tablet in this study, and IVR) on patient responses and potential measurement error associated with the PROMIS, PRO-CTCAE, and NRS within a variety of domains such as global health, physical function, social function, anxiety, depression, fatigue, sleep disturbance, and pain. The current study uses the randomized parallel groups design, in which each participant sees or hears each question only once because each patient answers the questionnaires using only one mode. This design overcomes possible memory effect. With regard to forced response vs. allowing patients to skip items, in many applications of electronic data capture, missing is not allowed. In PSAQ, respondents cannot be forced to respond to every item. In order to minimize features not intrinsic to modes, we opted to forego forced response in the IVR and CSAQ and allowed patients' nonresponse at the item level for all modes. Allowing research participants to skip questions they don't wish to answer is also consistent with our IRB's position on questionnairebased research.

\section{Methods}

\section{Sample}

This study is a part of a larger study whose primary aim was to assess the convergent validity of PROMIS, PROCTCAE, and NRS by comparing item responses for two groups based on ECOG PS (0-1 vs. 2-4). A secondary analysis was to assess the relationship between survival status and PRO scores. In order to achieve power for all primary and secondary analyses, the sample size for the primary study was based on a superiority analysis of survival between high and low PRO score groups. In the current equivalence study comparing modes of administration, we claim equivalence when the confidence interval of the difference in outcomes between modes is within a predetermined equivalence margin that represents a clinically acceptable range of difference. Using $\sigma$ of 2 based on the normative data on overall QOL NRS that include cancer trial patients [17], $\Delta$ of 0.45 corresponding to an effect size of 0.225 , which may start to be considered non-negligible on a $0-10$ scale, 2-sided type I error level of $5 \%$, and the sample size of 1184 , we obtain $94 \%$ statistical power. Using $\Delta$ of 0.40 corresponding to an effect size of 0.20 , we obtain $86 \%$ statistical power.

There were five participating sites (Mayo Clinic, M.D. Anderson, Memorial-Sloan-Kettering, Northwestern University, and University of North Carolina). Patients with a diagnosis of cancer who were initiating active anti-cancer treatment within the next seven days, were currently receiving anti-cancer treatment, or underwent surgery for cancer treatment in the past 14 days, were recruited in-person by research study associates/data managers when arriving at a participating institution for a cancer-related appointment. Patients were accrued from the main hospital sites and satellite clinics for each institution. Eligibility criteria included adults who possess the ability to use and understand the informed consent and privacy protection documentation (written in English) and interact with the data collection modes (i.e., read and answer questions on a computer screen, listen to questions and respond using an IVR telephone system, or fill out a paper questionnaire). Each eligible patient provided informed consent. Enrollment and distribution of accrual across disease groups and institutions were facilitated by a recruitment coordinator.

The resulting sample were randomized to PSAQ $(n=604)$, CSAQ $(n=603)$, or IVR $(n=602)$. Participants were asked to complete the questionnaires while at the clinic for their visit. A study coordinator handed the participant a folded paper questionnaire booklet (PSAQ arm), an iPad tablet computer (CSAQ arm) or directed the patient to a landline (i.e., hardwired to a telephone jack) telephone with a keypad (IVR arm). Twenty-eight patients across three arms did not respond at all, and one person switched from IVR to PSAQ. Excluding these patients, we analyzed the remaining 595 patients who received paper, 596 IVR, and 589 CSAQ.

\section{Measures}

We used PROMIS short forms and analogous NRS and PRO-CTCAE single-item rating scales. The PROMIS domains included in the study were emotional distressanxiety, emotional distress-depression, fatigue, pain interference, pain intensity, physical function, satisfaction with social roles, sleep disturbance, global mental health, and global physical health. We administered nine version 1.0 short forms derived from PROMIS item banks: Anxiety $8 \mathrm{a}$, Depression $8 \mathrm{a}$, Fatigue $7 \mathrm{a}$ with two added items from another fatigue short form, Sleep Disturbance 8a, 
Pain Intensity 3a, Pain Interference 8a, Ability to participate in Social Roles and Activities 8a, Global Mental Health, Global Physical Health, and Physical Function 10a. The PROMIS scores are on T-score scale, which we used for comparing the average scores between modes, and we did not transform the T-scores to 0-100 scale.

National Cancer Institute (NCI)'s PRO-CTCAE is a pool of adverse symptom items for patient self-reporting in NCI-sponsored clinical trials. The CTCAE is an existing lexicon of clinician-reported adverse event items required for use in all NCI-sponsored trials. Patient versions of CTCAE symptom items is intended to provide clinicians with more comprehensive information about the patient experience with treatment when trials are completed and reported. The PRO-CTCAE item bank consists of five "types" of items (present/not present, frequency, severity, interference with usual or daily activities, and amount of symptom). In this study, we included frequency, severity and interference items for PROCTCAE. The response options were never, rarely, occasionally, frequently, and almost constantly for frequency; none, mild, moderate, severe, and very severe for severity; and not at all, a little bit, somewhat, quite a bit, and very much for interference items.

We used NRS items for overall health-related QOL, five major QOL domains (e.g., sleep, pain, anxiety, depression, and fatigue), and items for each domain for which a PROMIs measure exists (e.g., social and physical function). NRS scores and PRO-CTCAE scores are on 0-10 and 1-5 integer rating scales respectively. For comparing mean scores, NRS and PRO-CTCAE item scores were linearly transformed to $0-100$ scales with higher scores indicating more of the construct in question (e.g., more fatigue, better physical function). Respondents answered 62 PROMIS items, 16 PRO-CTCAE items, and 11 NRS items.

Health literacy was measured by an item, "how confident are you filling out medical form by yourself?" Based on the findings by Chew et al. [18] of the screening threshold that optimizes both sensitivity and specificity, "Extremely" and "quite a bit" were coded as having adequate health literacy, and "somewhat", "a little bit", and "not at all" were coded as not having adequate health literacy.

\section{Measurement equivalence or lack of differential item functioning}

A critical step before using instruments to compare scores from different modes of administration is determining whether items have the same meaning to members of different groups [19]. Psychometric concern for measurement equivalence arises whenever group comparisons on observable scores are the focus of research [20]. Once measurement equivalence between modes of administration has been established, quantitative crossmode comparisons can be meaningfully conducted.

The R software package, lordif [21], was used to evaluate differential item functioning (DIF) in each of the PROMIS scales. Item-level data for all three types of measures (i.e., PROMIS, PRO-CTCAE, and NRS) were entered for each construct tested. Lordif assesses DIF using a hybrid of ordinal logistic regression and IRT framework. The main objective of fitting an IRT model under lordif is to obtain IRT trait estimates to serve as matching criterion. We tested whether the combined item set is unidimensional by conducting confirmatory factor analyses (CFA) treating the items as ordinal and using WLSMV estimator with lavaan $\mathrm{R}$ package [22]. Model fit was evaluated based on the Comparative Fit Index (CFI $\geq 0.95$ very good fit) and the Standardized Root Mean Square Error Residual $(S R M R \leq 0.08)$ [23]. We also estimated the proportion of total variance attributable to a general factor (i.e., coefficient omega, $\omega_{h}$ ) [24]: Values of 0.70 or higher suggest that the item set is sufficiently unidimensional for most analytic procedures that assume unidimensionality [25] McFadden pseudo $R^{2}$ change criterion of $\geq 0.02$ was used to flag items for DIF [26]. A value of pseudo $R^{2}$ less than 0.02 indicates a lack of evidence of differential interpretation of an item across modes.

\section{Comparison of means by modes of administration}

If no DIF is found, then we can meaningfully interpret the differences in scores between modes. If differences in average scores between modes are observed, then we can conclude that these differences come purely from the characteristics of the modes rather than DIF. If DIF is found for a certain mode in a given domain, we would score patients using newly derived item parameters for that mode before conducting mean comparisons between modes.

We compared the percentages of missing values among modes using the equality of proportion test with $X^{2}$ test statistic to ensure missing values are not driving differences across the modes. For constructs where lack of DIF was established, we concluded equivalence across modes if the margin of small effect size, defined as $\pm 0.20 \times$ pooled SD, completely surrounds $95 \%$ confidence intervals for difference in mean score. Here, one fifth of the pooled standard deviation indicates a small difference, following the observation by Coons et al. [27] that a "small" effect size difference is between 0.20 SD and 
Table 1 Patient characteristics by mode of administration

\begin{tabular}{|c|c|c|c|c|}
\hline Characteristic & PSAQ & CSAQ & IVR & $p$ value \\
\hline Sample size & 595 & 596 & 589 & \\
\hline Age (in years), mean (standard deviation) & $57(12)$ & $56(13)$ & $56(13)$ & .83 \\
\hline Gender, female, \% & 62 & 60 & 63 & .52 \\
\hline Race, Non-White, \% & 27 & 24 & 26 & .54 \\
\hline Ethnicity, Hispanic, \% & 4 & 8 & 5 & .03 \\
\hline Site, $\%$ & & & & .71 \\
\hline MD Anderson & 19 & 19 & 19 & \\
\hline Mayo Clinic & 47 & 48 & 46 & \\
\hline Memorial-Sloan Kettering & 8 & 8 & 8 & \\
\hline Northwestern University & 23 & 24 & 24 & \\
\hline University of North Carolina & 3 & 1 & 3 & \\
\hline Highest level of education, \% & & & & .83 \\
\hline High School graduate or lower & 29 & 29 & 28 & \\
\hline Vocational school degree, some college or college graduate & 53 & 55 & 53 & \\
\hline Graduate or professional school degree & 17 & 15 & 18 & \\
\hline Other & 1 & 1 & 1 & \\
\hline Marital status, married or marriage-like relationship, $\%$ & 72 & 68 & 71 & .35 \\
\hline Employment status, \% & & & & .12 \\
\hline Employed (full or part time) & 29 & 33 & 35 & \\
\hline On sick leave or disability & 27 & 27 & 23 & \\
\hline Other & 44 & 40 & 43 & \\
\hline Disease, \% & & & & .56 \\
\hline Breast & 27 & 24 & 28 & \\
\hline Lymphoma/myeloma & 18 & 23 & 22 & \\
\hline Prostate/bladder & 1 & 1 & 1 & \\
\hline Lung & 9 & 8 & 6 & \\
\hline Colorectal & 10 & 11 & 9 & \\
\hline Head/neck/gastroesophageal & 9 & 9 & 7 & \\
\hline Other & 26 & 25 & 26 & \\
\hline Disease stage, \% & & & & .46 \\
\hline । & 12 & 10 & 14 & \\
\hline$\|$ & 21 & 22 & 22 & \\
\hline III & 29 & 30 & 30 & \\
\hline IV & 38 & 38 & 35 & \\
\hline ECOG performance score, $\%$ & & & & .09 \\
\hline $0-1$ & 71 & 73 & 67 & \\
\hline $2-4$ & 29 & 27 & 33 & \\
\hline Cancer treatment in the past 2 weeks, $\%$ & & & & .09 \\
\hline Chemotherapy & 59 & 57 & 60 & \\
\hline Radiation & 4 & 2 & 2 & \\
\hline Surgery & 1 & 1 & 1 & \\
\hline Combination of above & 8 & 12 & 8 & \\
\hline None of the above & 29 & 27 & 30 & \\
\hline Current treatment intention, $\%$ & & & & .58 \\
\hline Curative & 70 & 70 & 72 & \\
\hline Palliative & 30 & 30 & 28 & \\
\hline Adequate confidence filling out medical forms, $\%$ & 85 & 81 & 84 & .23 \\
\hline
\end{tabular}

The percentages are percentages in each column for a given characteristic. PSAQ means paper-and-pencil self-administered questionnaire, CSAQ computerized self-administered questionnaire, and IVR questionnaire completed using interactive voice recording. Health literacy was measured by an item on confidence filling out medical form: "Extremely" and "quite a bit" were coded as having adequate confidence, and "somewhat", "a little bit", and "not at all" were coded as not having adequate confidence 
$0.49 \mathrm{SD}$ and that these values indicate minimal difference worthy of attention. If the $95 \%$ CI fell completely outside the margin, we concluded systematic score difference by modes. If the $95 \%$ CI partly overlaps the equivalence margin, we concluded neither equivalence nor difference.

\section{Results \\ Sample}

Patient characteristics by mode were similar, confirming successful randomization (Table 1). There were no significant differences in demographic characteristics among three modes: Distributions of age, proportions of male and female, proportion of non-white race, proportions of four different categories of education, being married, being employed or on sick leave, and having adequate health literacy had no statistically significant difference among modes of administration. In terms of medical characteristics, proportions of different types of cancers did not differ between modes as well as disease stage, ECOG performance score, types of cancer treatment in the past two weeks, and current treatment intention (i.e., curative or palliative). The only statistically significant difference in patient characteristics was found in proportion of Hispanic: $4 \%$ in PSAQ, $8 \%$ in CSAQ, and 5\% in IVR arm.

\section{Differential item functioning}

For the item sets combining PROMIS, NRS, and PROCTCAE, the CFA fit statistics based on CFI and SRMR were excellent for global physical health, global mental health, anxiety, depression, fatigue, sleep disturbance, pain intensity, pain interference, and ability to participate in social roles and activities. For physical function, CFI was 0.982 but SRMR was 0.126. For all constructs, $\omega_{\mathrm{h}}$ values exceeded 0.70 . For all constructs, no DIF of any kind was found for three modes (Table 2 and "Appendix 1"). All items in all analyses had a McFadden pseudo $R^{2}$ change below the criterion that indicates DIF $(<0.02)$. Because there was no item exhibiting DIF among PSAQ, CSAQ, and IVR, we did not have to let items take different item parameter values depending on the modes. Across methods, patients interpreted items in similar ways.

\section{Comparisons of scores based on modes}

Table 3 has the summary scores for each of the domains and modes. The average PROMIS T- scores indicated that the study population was not demonstrably different from the general population in most constructs. The exception was in physical function: Physical function PROMIS scores of our sample were 0.6 SDs lower compared to the general population. The average difference scores between modes are presented along with the margins of small effect size in Table 4 for ease of comparison. The scores on PSAQ and CSAQ were the most similar in that 29 out of 37 CI's of the mean differences were completely surrounded by the margins of small effect size, in which case we inferred equivalence. There were fewer results indicating equivalence between IVR

Table 2 Differential item functioning by modes of administration for anxiety

\begin{tabular}{|c|c|c|c|}
\hline Anxiety & $\begin{array}{l}\text { McFadden pseudo } R^{2} \\
\text { change }\left(R_{2}^{2}-R_{1}^{2}\right)\end{array}$ & $\begin{array}{l}\text { McFadden pseudo } R^{2} \\
\text { change }\left(R_{3}^{2}-R_{2}^{2}\right)\end{array}$ & $\begin{array}{l}\text { McFadden pseudo } \\
R^{2} \text { change }\left(R_{3}^{2}-R_{1}^{2}\right)\end{array}$ \\
\hline I felt fearful & 0.0001 & 0.0007 & 0.0005 \\
\hline I found it hard to focus on anything other than my anxiety & 0.0041 & 0.0051 & 0.0010 \\
\hline My worries overwhelmed me & 0.0019 & 0.0028 & 0.0008 \\
\hline I felt uneasy & 0.0007 & 0.0015 & 0.0008 \\
\hline I felt nervous & 0.0017 & 0.0023 & 0.0006 \\
\hline I felt like I needed help for my anxiety & 0.0015 & 0.0029 & 0.0014 \\
\hline I felt anxious & 0.0002 & 0.0004 & 0.0001 \\
\hline I felt tense & 0.0011 & 0.0015 & 0.0003 \\
\hline (NRS) describe the level of anxiety on average & 0.0005 & 0.0005 & 0.0000 \\
\hline (PRO-CTCAE) How often did you feel anxiety? & 0.0049 & 0.0058 & 0.0009 \\
\hline (PRO-CTCAE) What was the severity of your anxiety at the WORST? & 0.0025 & 0.0025 & 0.0000 \\
\hline (PRO-CTCAE) How much did anxiety interfere with usual/daily activities? & 0.0009 & 0.0017 & 0.0008 \\
\hline
\end{tabular}

A base model (model 1) posits that only the trait level predicts responses. A second model (model 2) has both trait level and group as independent variables. If model 2 predicts item responses statistically significantly better than model 1 (i.e., McFadden pseudo $R^{2}$ change $\left.\left(R_{2}^{2}-R_{1}^{2}\right) \geq 0.02\right)$, then there is uniform DIF. In uniform DIF, DIF has a consistent impact across trait levels. If the model that includes an interaction term between trait and group (model 3 ) fits significantly better than model 2 (i.e., McFadden pseudo $R^{2}$ change $\left.\left(R_{3}^{2}-R_{2}^{2}\right) \geq 0.02\right)$, then the impact of DIF varies by trait level (nonuniform DIF). If model 3 fits significantly better than model 1 (i.e., McFadden pseudo $R^{2}$ change $\left.\left(R_{3}^{2}-R_{1}^{2}\right) \geq 0.02\right)$, there is overall or total DIF 
Table 3 Health-related quality of life (HRQOL) scores by mode of administration

\begin{tabular}{|c|c|c|c|}
\hline Domain questionnaire and scale & PSAQ mean (SD) & CSAQ mean (SD) & IVR mean (SD) \\
\hline \multicolumn{4}{|l|}{ Global/general health } \\
\hline PROMIS global mental & $48.6(8.2)$ & $49.6(8.5)$ & $50.4(7.9)$ \\
\hline PROMIS global physical & $43.7(8.3)$ & $44.0(8.5)$ & $44.1(7.6)$ \\
\hline NRS overall QOL & $72.6(20.7)$ & $74.4(20.2)$ & $72.2(21.5)$ \\
\hline NRS emotional well-being & $75.7(21.0)$ & $77.1(19.4)$ & $75.3(20.4)$ \\
\hline NRS mental well-being & $80.2(19.6)$ & $81.1(18.4)$ & $78.8(19.7)$ \\
\hline \multicolumn{4}{|l|}{ Physical function } \\
\hline PROMIS physical function & $43.7(8.3)$ & $44.0(8.5)$ & $44.1(7.6)$ \\
\hline NRS physical well-being & $69.6(22.4)$ & $71.2(20.2)$ & $71.9(21.6)$ \\
\hline \multicolumn{4}{|l|}{ Social function } \\
\hline PROMIS social function & $48.5(9.6)$ & $49.0(9.6)$ & $49.2(8.8)$ \\
\hline NRS social activity & $66.0(26.0)$ & $68.5(24.1)$ & $69.6(23.9)$ \\
\hline \multicolumn{4}{|l|}{ Emotional distress_-anxiety } \\
\hline PROMIS anxiety & $50.2(9.8)$ & $49.3(9.6)$ & $49.2(9.2)$ \\
\hline NRS anxiety & $28.2(26.6)$ & $27.6(27.3)$ & $22.7(24.1)$ \\
\hline PRO-CTCAE anxiety frequency & $32.2(25.9)$ & $29.3(25.1)$ & $25.0(24.6)$ \\
\hline PRO-CTCAE anxiety severity & $27.7(24.1)$ & $25.0(23.1)$ & $22.0(22.2)$ \\
\hline PRO-CTCAE anxiety interference & $16.9(23.2)$ & $15.3(22.9)$ & $12.2(19.8)$ \\
\hline \multicolumn{4}{|l|}{ Emotional distress-depression } \\
\hline PROMIS depression & $48.0(9.1)$ & $47.4(8.9)$ & $48.1(7.8)$ \\
\hline NRS depression & $17.4(23.1)$ & $16.7(23.2)$ & $12.7(18.7)$ \\
\hline PRO-CTCAE cheer frequency & $16.4(22.5)$ & $15.7(22.8)$ & $12.7(19.5)$ \\
\hline PRO-CTCAE cheer severity & $14.3(22.0)$ & $13.0(20.8)$ & $10.4(17.4)$ \\
\hline PRO-CTCAE cheer interference & $12.4(20.9)$ & $10.7(20.1)$ & $9.0(17.1)$ \\
\hline PRO-CTCAE sad frequency & $29.4(23.8)$ & $27.7(24.0)$ & $24.3(20.4)$ \\
\hline PRO-CTCAE sad severity & $24.1(21.7)$ & $23.3(22.2)$ & $20.0(19.7)$ \\
\hline PRO-CTCAE sad interference & $14.8(21.8)$ & $13.6(21.7)$ & $11.4(18.6)$ \\
\hline \multicolumn{4}{|l|}{ Fatigue } \\
\hline PROMIS fatigue & $53.0(9.0)$ & $52.2(9.4)$ & $51.0(8.7)$ \\
\hline NRS fatigue & $40.7(26.0)$ & $39.5(27.1)$ & $33.9(24.6)$ \\
\hline PRO-CTCAE fatigue severity & $41.2(23.8)$ & $39.8(24.5)$ & $36.5(23.4)$ \\
\hline PRO-CTCAE fatigue interference & $36.7(26.8)$ & $36.3(27.7)$ & $31.8(24.8)$ \\
\hline \multicolumn{4}{|l|}{ Sleep } \\
\hline PROMIS sleep disturbance & $50.1(9.6)$ & $49.2(9.3)$ & $49.6(8.9)$ \\
\hline NRS sleep overall & $36.5(25.2)$ & $33.7(23.5)$ & $34.4(23.4)$ \\
\hline PRO-CTCAE sleep severity & $33.9(26.9)$ & $30.2(25.8)$ & $28.1(25.9)$ \\
\hline PRO-CTCAE sleep interference & $25.5(26.1)$ & $25.1(26.3)$ & $21.1(24.1)$ \\
\hline \multicolumn{4}{|l|}{ Pain } \\
\hline PROMIS pain intensity & $49.7(10.6)$ & $49.5(11.0)$ & $48.4(10.8)$ \\
\hline PROMIS pain interference & $51.8(9.9)$ & $51.3(10.0)$ & $50.7(9.5)$ \\
\hline NRS pain frequency & $29.3(29.4)$ & $27.8(28.7)$ & $26.0(28.7)$ \\
\hline NRS pain severity & $25.9(26.2)$ & $25.9(27.1)$ & $23.7(25.5)$ \\
\hline PRO-CTCAE pain frequency & $38.0(30.6)$ & $38.6(32.0)$ & $35.1(31.8)$ \\
\hline PRO-CTCAE pain severity & $32.0(26.8)$ & $32.1(27.9)$ & $30.2(28.4)$ \\
\hline PRO-CTCAE pain interference & $24.5(27.9)$ & $24.7(28.7)$ & $22.1(26.8)$ \\
\hline
\end{tabular}

PSAQ means paper-and-pencil self-administered questionnaire, CSAQ computerized self-administered questionnaire, and IVR questionnaire completed using interactive voice recording. The PROMIS scores were on a T-score metric, whereas NRS and PRO-CTCAE items were transformed to 0-100 scale 
Table 4 Differences in HRQOL scores and margins of small effect size by mode of administration

\begin{tabular}{|c|c|c|c|c|c|c|}
\hline $\begin{array}{l}\text { Domain questionnaire } \\
\text { and scale }\end{array}$ & $\begin{array}{l}\text { PSAQ-CSAQ mean diff } \\
(95 \% \mathrm{Cl})\end{array}$ & $\begin{array}{l}\text { Margin of } \\
\text { small effect } \\
\text { size }\end{array}$ & $\begin{array}{l}\text { CSAQ-IVR mean diff } \\
(95 \% \mathrm{CI})\end{array}$ & $\begin{array}{l}\text { Margin of } \\
\text { small effect } \\
\text { size }\end{array}$ & $\begin{array}{l}\text { IVR-PSAQ Mean diff } \\
(95 \% \mathrm{Cl})\end{array}$ & $\begin{array}{l}\text { Margin of } \\
\text { small effect } \\
\text { size }\end{array}$ \\
\hline \multicolumn{7}{|l|}{ Global/general health } \\
\hline PROMIS global mental & $-1.05(-2.01,-0.09)$ & \pm 1.67 & $-0.77(-1.71,0.17)$ & \pm 1.64 & $1.82(0.89,2.75)$ & \pm 1.62 \\
\hline PROMIS global physical & $-0.41(-1.39,0.57)$ & \pm 1.70 & $-0.03(-1.00,0.94)$ & \pm 1.69 & $0.44(-0.52,1.41)$ & \pm 1.67 \\
\hline NRS overall QOL & $-1.76(-4.10,0.58)$ & \pm 4.09 & $2.19(-0.20,4.58)$ & \pm 4.17 & $-0.43(-2.85,1.99)$ & \pm 4.22 \\
\hline $\begin{array}{l}\text { NRS emotional well- } \\
\text { being }\end{array}$ & $-1.44(-3.75,0.88)$ & \pm 4.05 & $1.82(-0.46,4.10)$ & \pm 3.99 & $-0.38(-2.76,1.99)$ & \pm 4.15 \\
\hline NRS mental well-being & $-0.88(-3.06,1.29)$ & \pm 3.80 & $2.24(0.06,4.42)$ & \pm 3.81 & $-1.36(-3.61,0.89)$ & \pm 3.92 \\
\hline \multicolumn{7}{|l|}{ Physical function } \\
\hline $\begin{array}{l}\text { PROMIS physical func- } \\
\text { tion }\end{array}$ & $-0.39(-1.35,0.58)$ & \pm 1.68 & $-0.05(-0.98,0.88)$ & \pm 1.61 & $0.43(-0.48,1.35)$ & \pm 1.60 \\
\hline NRS Physical well-being & $-1.55(-3.99,0.90)$ & \pm 4.26 & $-0.72(-3.15,1.70)$ & \pm 4.18 & $2.27(-0.27,4.82)$ & \pm 4.41 \\
\hline \multicolumn{7}{|l|}{ Social function } \\
\hline PROMIS social function & $-0.51(-1.61,0.59)$ & \pm 1.92 & $-0.22(-1.28,0.84)$ & \pm 1.84 & $0.73(-0.34,1.79)$ & \pm 1.85 \\
\hline NRS social activity & $-2.43(-5.32,0.46)$ & \pm 5.01 & $-1.16(-3.95,1.62)$ & \pm 4.80 & $3.59(0.70,6.48)$ & \pm 4.99 \\
\hline \multicolumn{7}{|l|}{$\begin{array}{l}\text { Emotional distress- } \\
\text { anxiety }\end{array}$} \\
\hline PROMIS anxiety & $0.77(-0.35,1.88)$ & \pm 1.94 & $0.21(-0.88,1.30)$ & \pm 1.89 & $-0.98(-2.08,0.12)$ & \pm 1.90 \\
\hline NRS anxiety & $0.60(-2.51,3.70)$ & \pm 5.40 & $5.01(2.02,7.99)$ & \pm 5.16 & $-5.60(-8.54,-2.66)$ & \pm 5.08 \\
\hline $\begin{array}{l}\text { PRO-CTCAE anxiety } \\
\text { frequency }\end{array}$ & $2.89(-0.04,5.83)$ & \pm 5.10 & $4.36(1.48,7.23)$ & \pm 4.97 & $-7.25(-10.2,-4.33)$ & \pm 5.05 \\
\hline $\begin{array}{l}\text { PRO-CTCAE anxiety } \\
\text { severity }\end{array}$ & $2.66(-0.05,5.37)$ & \pm 4.72 & $3.09(0.47,5.72)$ & \pm 4.53 & $-5.76(-8.44,-3.07)$ & \pm 4.64 \\
\hline $\begin{array}{l}\text { PRO-CTCAE anxiety } \\
\text { interference }\end{array}$ & $1.62(-1.03,4.27)$ & \pm 4.62 & $3.10(0.62,5.58)$ & \pm 4.28 & $-4.72(-7.22,-2.22)$ & \pm 4.32 \\
\hline \multicolumn{7}{|l|}{$\begin{array}{l}\text { Emotional distress- } \\
\text { depression }\end{array}$} \\
\hline PROMIS depression & $0.57(-0.47,1.60)$ & \pm 1.80 & $-0.56(-1.53,0.41)$ & \pm 1.68 & $-0.01(-0.99,0.97)$ & \pm 1.70 \\
\hline NRS depression & $0.75(-1.92,3.42)$ & \pm 4.63 & $3.95(1.52,6.39)$ & \pm 4.21 & $-4.70(-7.14,-2.27)$ & \pm 4.21 \\
\hline $\begin{array}{l}\text { PRO-CTCAE cheer } \\
\text { Frequency }\end{array}$ & $0.62(-1.99,3.23)$ & \pm 4.53 & $3.05(0.59,5.51)$ & \pm 4.25 & $-3.67(-6.11,-1.23)$ & \pm 4.22 \\
\hline $\begin{array}{l}\text { PRO-CTCAE cheer } \\
\text { severity }\end{array}$ & $1.31(-1.17,3.78)$ & \pm 4.29 & $2.66(0.43,4.89)$ & \pm 3.84 & $-3.97(-6.27,-1.66)$ & \pm 3.97 \\
\hline $\begin{array}{l}\text { PRO-CTCAE cheer } \\
\text { interference }\end{array}$ & $1.73(-0.64,4.09)$ & \pm 4.11 & $1.72(-0.44,3.89)$ & \pm 3.73 & $-3.45(-5.67,-1.23)$ & \pm 3.83 \\
\hline $\begin{array}{l}\text { PRO-CTCAE sad fre- } \\
\text { quency }\end{array}$ & $1.66(-1.10,4.41)$ & \pm 4.78 & $3.48(0.88,6.07)$ & \pm 4.47 & $-5.13(-7.71,-2.55)$ & \pm 4.44 \\
\hline PRO-CTCAE sad severity & $0.78(-1.75,3.30)$ & \pm 4.38 & $3.35(0.91,5.78)$ & \pm 4.20 & $-4.12(-6.53,-1.72)$ & \pm 4.15 \\
\hline $\begin{array}{l}\text { PRO-CTCAE sad interfer- } \\
\text { ence }\end{array}$ & $1.14(-1.36,3.64)$ & \pm 4.34 & $2.30(-0.04,4.64)$ & \pm 4.04 & $-3.44(-5.79,-1.09)$ & \pm 4.05 \\
\hline \multicolumn{7}{|l|}{ Fatigue } \\
\hline PROMIS fatigue & $0.72(-0.34,1.77)$ & \pm 1.84 & $1.31(0.25,2.36)$ & \pm 1.82 & $-2.02(-3.05,-1.00)$ & \pm 1.77 \\
\hline NRS fatigue & $1.17(-1.89,4.22)$ & \pm 5.32 & $5.69(2.68,8.69)$ & \pm 5.19 & $-6.85(-9.80,-3.91)$ & \pm 5.07 \\
\hline $\begin{array}{l}\text { PRO-CTCAE fatigue } \\
\text { severity }\end{array}$ & $1.38(-1.39,4.15)$ & \pm 4.83 & $3.34(0.56,6.12)$ & \pm 4.79 & $-4.72(-7.46,-1.98)$ & \pm 4.72 \\
\hline $\begin{array}{l}\text { PRO-CTCAE fatigue } \\
\text { interference }\end{array}$ & $0.47(-2.66,3.60)$ & \pm 5.46 & $4.55(1.50,7.60)$ & \pm 5.27 & $-5.02(-8.02,-2.02)$ & \pm 5.17 \\
\hline \multicolumn{7}{|l|}{ Sleep } \\
\hline $\begin{array}{l}\text { PROMIS sleep distur- } \\
\text { bance }\end{array}$ & $0.90(-0.19,1.98)$ & \pm 1.90 & $-0.35(-1.40,0.71)$ & \pm 1.82 & $-0.55(-1.62,0.53)$ & \pm 1.86 \\
\hline NRS sleep overall & $2.79(-0.00,5.59)$ & \pm 4.88 & $-0.64(-3.36,2.08)$ & \pm 4.69 & $-2.15(-4.97,0.67)$ & \pm 4.87 \\
\hline $\begin{array}{l}\text { PRO-CTCAE sleep } \\
\text { severity }\end{array}$ & $3.70(0.69,6.72)$ & \pm 5.26 & $2.11(-0.88,5.09)$ & \pm 5.16 & $-5.81(-8.86,-2.76)$ & \pm 5.28 \\
\hline
\end{tabular}


Table 4 (continued)

\begin{tabular}{|c|c|c|c|c|c|c|}
\hline $\begin{array}{l}\text { Domain questionnaire } \\
\text { and scale }\end{array}$ & $\begin{array}{l}\text { PSAQ-CSAQ mean diff } \\
(95 \% \mathrm{CI})\end{array}$ & $\begin{array}{l}\text { Margin of } \\
\text { small effect } \\
\text { size }\end{array}$ & $\begin{array}{l}\text { CSAQ-IVR mean diff } \\
(95 \% \mathrm{Cl})\end{array}$ & $\begin{array}{l}\text { Margin of } \\
\text { small effect } \\
\text { size }\end{array}$ & $\begin{array}{l}\text { IVR-PSAQ Mean diff } \\
(95 \% \mathrm{CI})\end{array}$ & $\begin{array}{l}\text { Margin of } \\
\text { small effect } \\
\text { size }\end{array}$ \\
\hline $\begin{array}{l}\text { PRO-CTCAE sleep inter- } \\
\text { ference }\end{array}$ & $0.39(-2.62,3.39)$ & \pm 5.23 & $4.12(1.20,7.04)$ & \pm 5.05 & $-4.50(-7.41,-1.60)$ & \pm 5.02 \\
\hline \multicolumn{7}{|l|}{ Pain } \\
\hline PROMIS pain intensity & $0.16(-1.07,1.40)$ & \pm 2.16 & $1.05(-0.20,2.31)$ & \pm 2.18 & $-1.22(-2.45,0.02)$ & \pm 2.14 \\
\hline $\begin{array}{l}\text { PROMIS pain interfer- } \\
\text { ence }\end{array}$ & $0.56(-0.58,1.70)$ & \pm 1.99 & $0.62(-0.51,1.75)$ & \pm 1.95 & $-1.18(-2.30,-0.06)$ & \pm 1.94 \\
\hline NRS pain frequency & $1.53(-1.80,4.85)$ & \pm 5.81 & $1.79(-1.53,5.11)$ & \pm 5.74 & $-3.32(-6.68,0.04)$ & \pm 5.81 \\
\hline NRS pain severity & $-0.02(-3.07,3.03)$ & \pm 5.33 & $2.29(-0.76,5.34)$ & \pm 5.26 & $-2.27(-5.26,0.72)$ & \pm 5.17 \\
\hline $\begin{array}{l}\text { PRO-CTCAE pain } \\
\text { frequency }\end{array}$ & $-0.56(-4.15,3.02)$ & \pm 6.26 & $3.60(-0.09,7.29)$ & \pm 6.37 & $-3.04(-6.64,0.57)$ & \pm 6.24 \\
\hline $\begin{array}{l}\text { PRO-CTCAE pain } \\
\text { severity }\end{array}$ & $-0.12(-3.25,3.01)$ & \pm 5.47 & $1.96(-1.30,5.22)$ & \pm 5.63 & $-1.83(-5.02,1.36)$ & \pm 5.52 \\
\hline $\begin{array}{l}\text { PRO-CTCAE pain inter- } \\
\text { ference }\end{array}$ & $-0.17(-3.41,3.08)$ & \pm 5.66 & $2.68(-0.54,5.89)$ & \pm 5.56 & $-2.51(-5.67,0.66)$ & \pm 5.48 \\
\hline
\end{tabular}

Results in bold text indicate where an upper or lower confidence limit exceeds the margin of small effect size. PSAQ means paper-and-pencil self-administered questionnaire, CSAQ computerized self-administered questionnaire, and IVR questionnaire completed using interactive voice recording. The PROMIS scores were on a T-score metric, whereas NRS and PRO-CTCAE items were transformed to 0-100 scale

and other modes: 14 out of 37 CI's for CSAQ-IVR and 9 CI's for PSAQ-IVR were within the margin of small effect sizes. For 95\% CI's that were not within the margin of small effect sizes, none of the CI's were completely outside the margins, which was somewhat inconclusive in that it indicates neither equivalence nor difference. In some instances, the observed point estimate of outcome difference lied outside the equivalence margins, which indicated a clearer lack of equivalence. For example, the difference between CSAQ and IVR on NRS fatigue, 5.69, was outside the equivalence margin of $[-5.19,5.19]$. There were more of such differences between IVR and PSAQ in NRS anxiety, NRS depression, NRS fatigue, PRO-CTCAE anxiety items, a PRO-CTCAE item asking the frequency of feeling sadness, and a PRO-CTCAE item asking the severity of sleep difficulty. In general, those who responded on IVR reported higher function and lower symptoms compared to other modes, and this trend was most marked in IVR-PSAQ comparison.

Although IVR mode tended to elicit slightly higher patient-reported function and lower symptoms, there were some inconsistencies. For example, IVR scores on PROMIS global mental health were higher (despite small effect size), whereas for NRS, those in IVR arm reported lower QOL and mental/ emotional well-being items compared to CSAQ. We investigated whether this could be attributed to possible primacy (choosing the first option) on NRS items in IVR given the auditory nature of IVR
("Appendix 2"). Across all the single-item measures, after Bonferroni correction, there was a statistically significant primacy effect for NRS overall QOL in the IVR mode. In addition, there were two comparisons (i.e., (1) NRS emotional well-being and (2) NRS mental well-being) in which patients in the PSAQ and CSAQ were more likely to choose 10 compared to those in the IVR arm; thus, suggesting a recency effect (choosing the last option) for PSAQ and CSAQ.

Percentages of missing values were comparable for PSAQ and CSAQ modes. However, percentages of missing values were slightly higher for IVR compared to PSAQ and CSAQ modes (Table 5). For multi-item scales (i.e., PROMIS short forms), we investigated whether some of the higher missing values for IVR could be due to fatigue and patients choosing not to respond to items that appear at the end of each scale. However, as "Appendix 3" shows, the percentages of missing values within each multi-item measure tended to be stable from the beginning to the end of each scale. In addition, the patients who did not respond to one scale tended to consistently not respond to other scales. Figure 1 shows that patients did not report more difficulty with IVR. All sites had more missingness from IVR than other modes. Some of these sites reported random technical problems of some data not being saved behind the scenes that patients were not aware of. 
Table 5 Percentage of missing values in scores by mode of administration for patients with baseline data

\begin{tabular}{|c|c|c|c|c|c|c|}
\hline Domain questionnaire and scale & $\begin{array}{l}\text { \% Missing } \\
\text { PSAQ }\end{array}$ & $\begin{array}{l}\text { \% Missing } \\
\text { CSAQ }\end{array}$ & $\%$ Missing IVR & $\mathrm{p}$ value all groups & $\begin{array}{l}p \text { value PSAQ } \\
\text { vs CSAQ }\end{array}$ & $\begin{array}{l}\text { p value IVR } \\
\text { vs others }\end{array}$ \\
\hline \multicolumn{7}{|l|}{ Global/general health } \\
\hline PROMIS global mental & 2.8 & 0.7 & 2.3 & .0201 & .0050 & .4151 \\
\hline PROMIS global physical & 2.6 & 0.5 & 2.5 & .0103 & .0031 & .0009 \\
\hline NRS overall QOL & 1.4 & 0.5 & 2.5 & .0166 & .1119 & .0198 \\
\hline NRS emotional well-being & 1.4 & 0.3 & 2.5 & .0068 & .0482 & .0119 \\
\hline NRS mental well-being & 1.7 & 0.3 & 2.7 & .0053 & .0186 & .0170 \\
\hline \multicolumn{7}{|l|}{ Physical function } \\
\hline PROMIS physical function & 1.2 & 1.2 & 3.7 & .0021 & .9290 & .0009 \\
\hline NRS physical well-being & 1.4 & 1.5 & 5.7 & $<.0001$ & .8513 & $<.0001$ \\
\hline \multicolumn{7}{|l|}{ Social function } \\
\hline PROMIS social function & 1.7 & 1.0 & 5.2 & $<.0001$ & .2983 & $<.0001$ \\
\hline NRS social activity & 2.6 & 2.5 & 5.2 & .0173 & .9147 & .0069 \\
\hline \multicolumn{7}{|l|}{ Emotional distress - anxiety } \\
\hline PROMIS anxiety & 2.0 & 1.0 & 5.2 & $<.0001$ & .1535 & $<.0001$ \\
\hline NRS anxiety & 2.0 & 1.5 & 5.4 & .0001 & .5133 & $<.0001$ \\
\hline PRO-CTCAE anxiety frequency & 2.0 & 1.5 & 5.4 & .0001 & .5133 & $<.0001$ \\
\hline PRO-CTCAE anxiety severity & 2.2 & 1.4 & 5.4 & $<.0001$ & .2782 & $<.0001$ \\
\hline PRO-CTCAE anxiety interference & 2.3 & 1.2 & 5.4 & $<.0001$ & .1298 & $<.0001$ \\
\hline \multicolumn{7}{|l|}{ Emotional distress-depression } \\
\hline PROMIS depression & 2.5 & 1.4 & 4.8 & .0012 & .1517 & .0008 \\
\hline NRS depression & 2.8 & 1.9 & 4.8 & .0109 & .2812 & .0068 \\
\hline CTC cheer frequency & 2.6 & 1.9 & 4.8 & .0088 & .3611 & .0047 \\
\hline CTC Cheer severity & 3.3 & 2.0 & 6.2 & .0006 & .1822 & .0004 \\
\hline CTC cheer interference & 3.0 & 1.7 & 6.0 & .0002 & .1460 & .0001 \\
\hline CTC sad frequency & 3.0 & 1.7 & 6.0 & .0002 & .1460 & .0001 \\
\hline CTC sad severity & 2.5 & 1.5 & 6.0 & $<.0001$ & .2324 & $<.0001$ \\
\hline CTC sad interference & 2.5 & 1.7 & 6.0 & $<.0001$ & .3349 & $<.0001$ \\
\hline \multicolumn{7}{|l|}{ Fatigue } \\
\hline PROMIS fatigue & 1.9 & 0.7 & 6.0 & $<.0001$ & .0660 & $<.0001$ \\
\hline NRS fatigue & 2.0 & 1.2 & 6.2 & $<.0001$ & .2476 & $<.0001$ \\
\hline PRO-CTCAE fatigue severity & 2.0 & 0.8 & 6.4 & $<.0001$ & .0866 & $<.0001$ \\
\hline PRO-CTCAE fatigue interference & 2.0 & 0.8 & 6.5 & $<.0001$ & .0866 & $<.0001$ \\
\hline \multicolumn{7}{|l|}{ Sleep } \\
\hline PROMIS sleep disturbance & 0.8 & 0.5 & 6.2 & $<.0001$ & .5581 & $<.0001$ \\
\hline NRS sleep overall & 1.2 & 0.8 & 6.4 & $<.0001$ & .4976 & $<.0001$ \\
\hline PRO-CTCAE sleep severity & 1.4 & 0.7 & 5.9 & $<.0001$ & .2163 & $<.0001$ \\
\hline PRO-CTCAE sleep interference & 1.9 & 1.0 & 5.7 & $<.0001$ & .2155 & $<.0001$ \\
\hline \multicolumn{7}{|l|}{ Pain } \\
\hline PROMIS pain intensity & 0.8 & 0.7 & 5.7 & $<.0001$ & .8393 & $<.0001$ \\
\hline PROMIS pain interference & 0.9 & 0.8 & 5.9 & $<.0001$ & .8753 & $<.0001$ \\
\hline NRS pain frequency & 1.1 & 0.8 & 5.9 & $<.0001$ & .6686 & $<.0001$ \\
\hline NRS pain severity & 0.9 & 1.2 & 5.9 & $<.0001$ & .6613 & $<.0001$ \\
\hline PRO-CTCAE pain frequency & 0.9 & 1.0 & 5.9 & $<.0001$ & .8786 & $<.0001$ \\
\hline PRO-CTCAE pain severity & 1.2 & 0.8 & 5.9 & $<.0001$ & .4976 & $<.0001$ \\
\hline PRO-CTCAE pain interference & 1.1 & 0.8 & 5.9 & $<.0001$ & .6686 & $<.0001$ \\
\hline
\end{tabular}

The equality of proportion tests with $x^{2}$ test statistic were used to derive the $p$ values. Because of multiple comparison concerns, $p<.0001$ will be used as a guideline to identify likely significant differences 


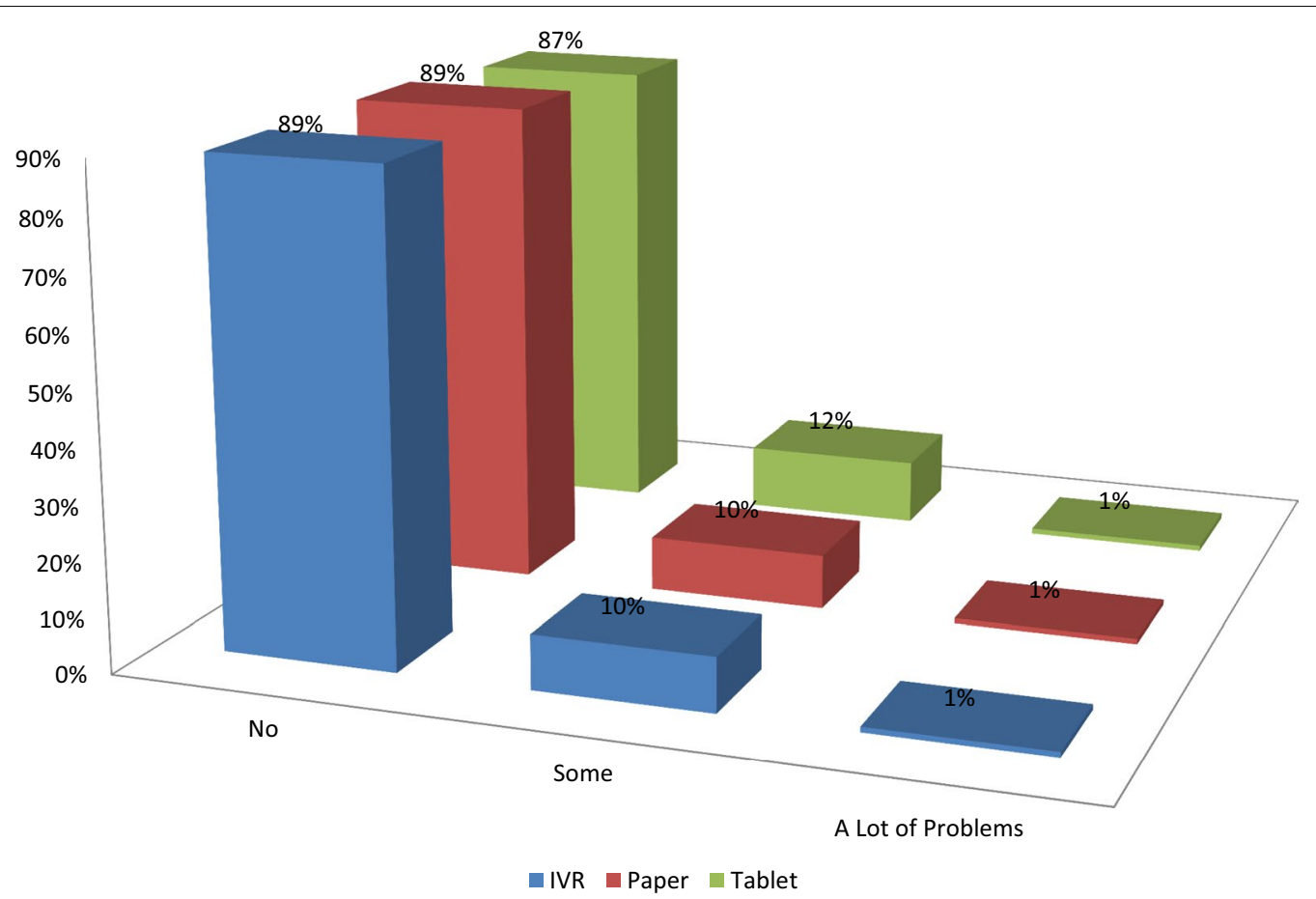

Fig. 1 Any problems using survey today?

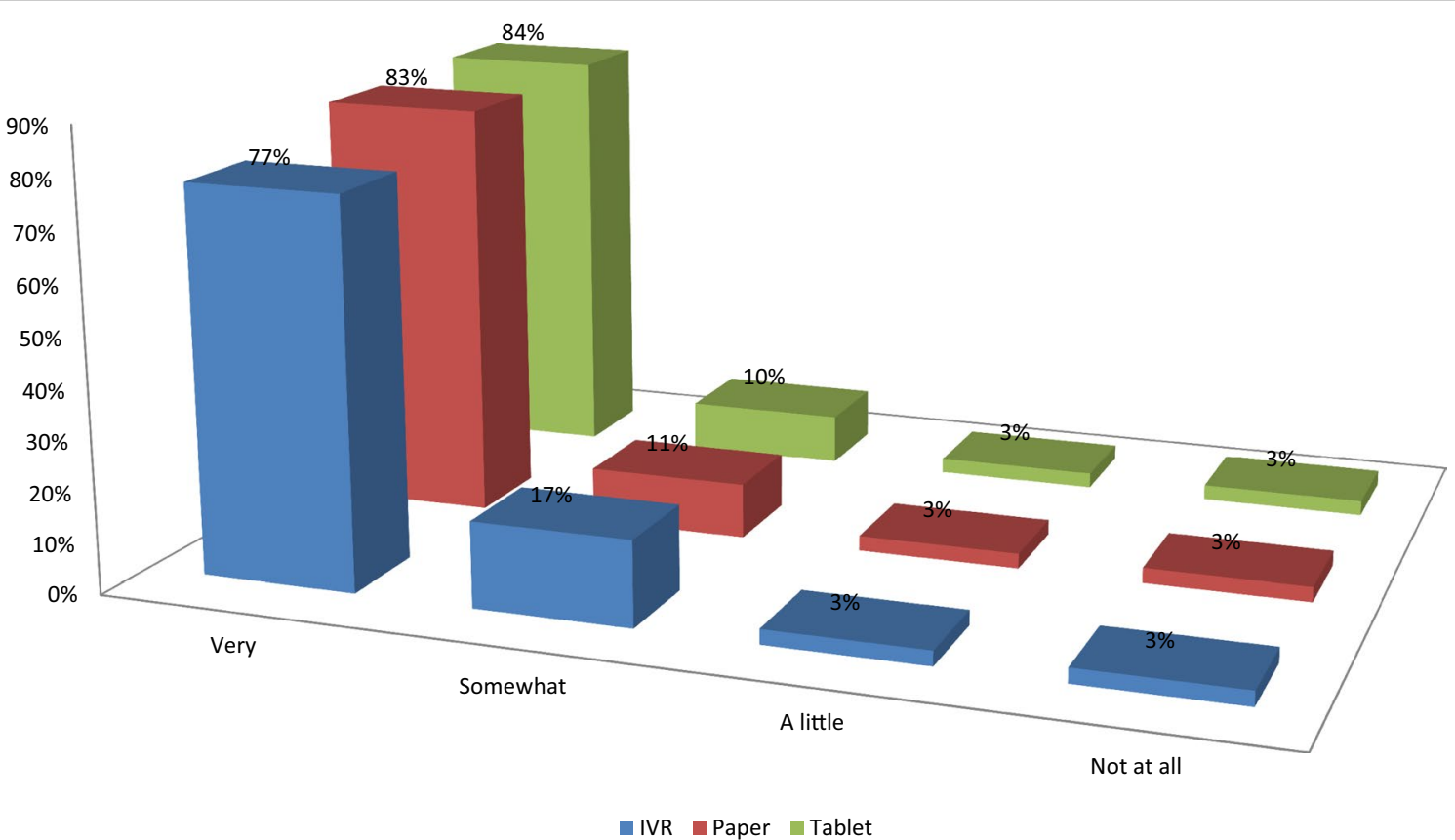

Fig. 2 How comfortable were you when using the survey today? 


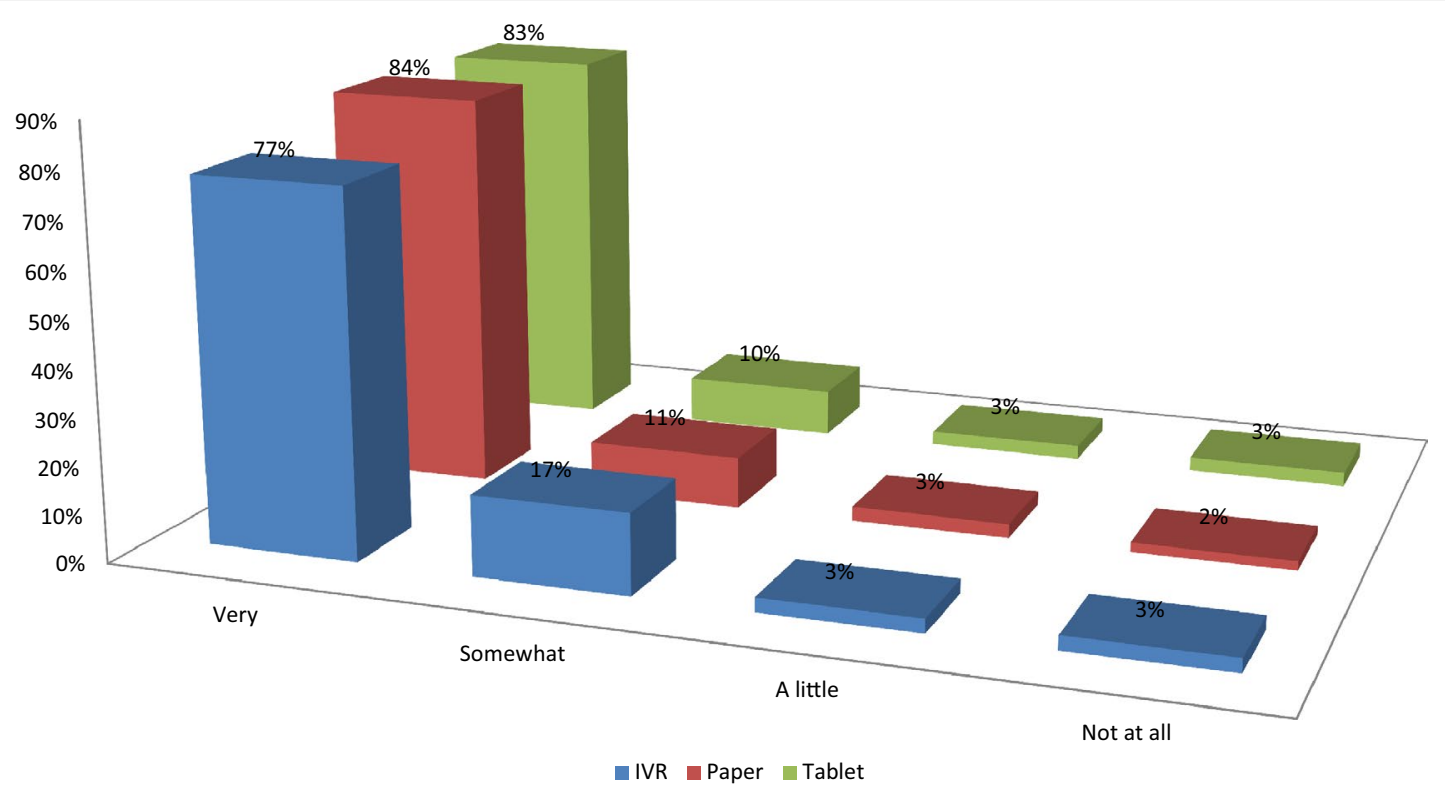

Fig. 3 How willing to use paper survey?

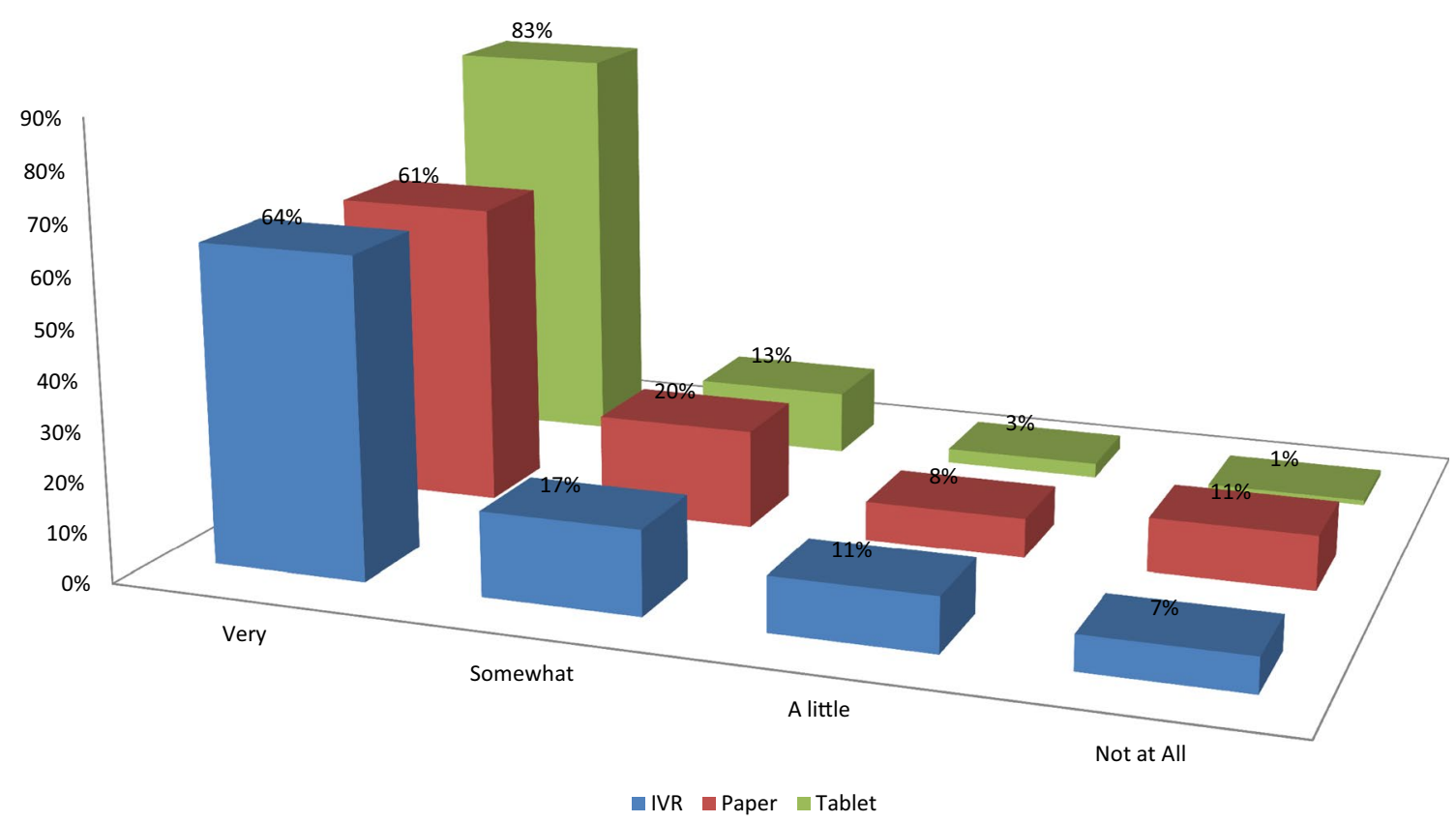

Fig. 4 How willing to use touchscreen computer or tablet survey?

\section{Patient preferences for three modes}

The proportions of patients reporting that they were very comfortable answering surveys were about 6-7\% lower for IVR than PSAQ and CSAQ (Fig. 2). Patients who were randomized to PSAQ (84\%) and CSAQ (83\%) were more likely to say that they are very willing to use PSAQ in the future (Fig. 3); The patients who were randomized to IVR were less likely (6-7\% less likely) to respond that they are very willing to use PSAQ. Patients who were randomized to CSAQ were most likely to respond that they are very 


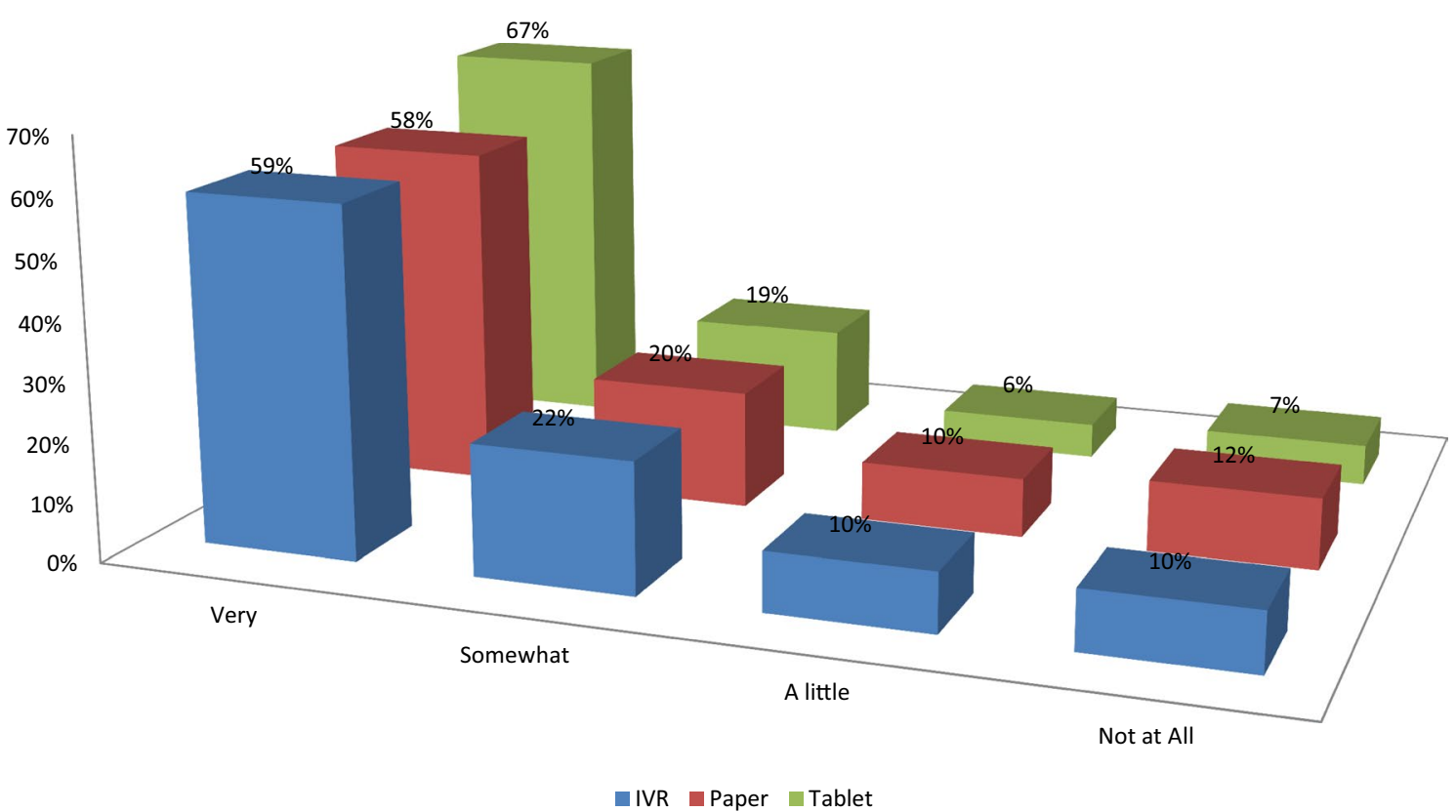

Fig. 5 Willing to use regular computer with keyboard/mouse?

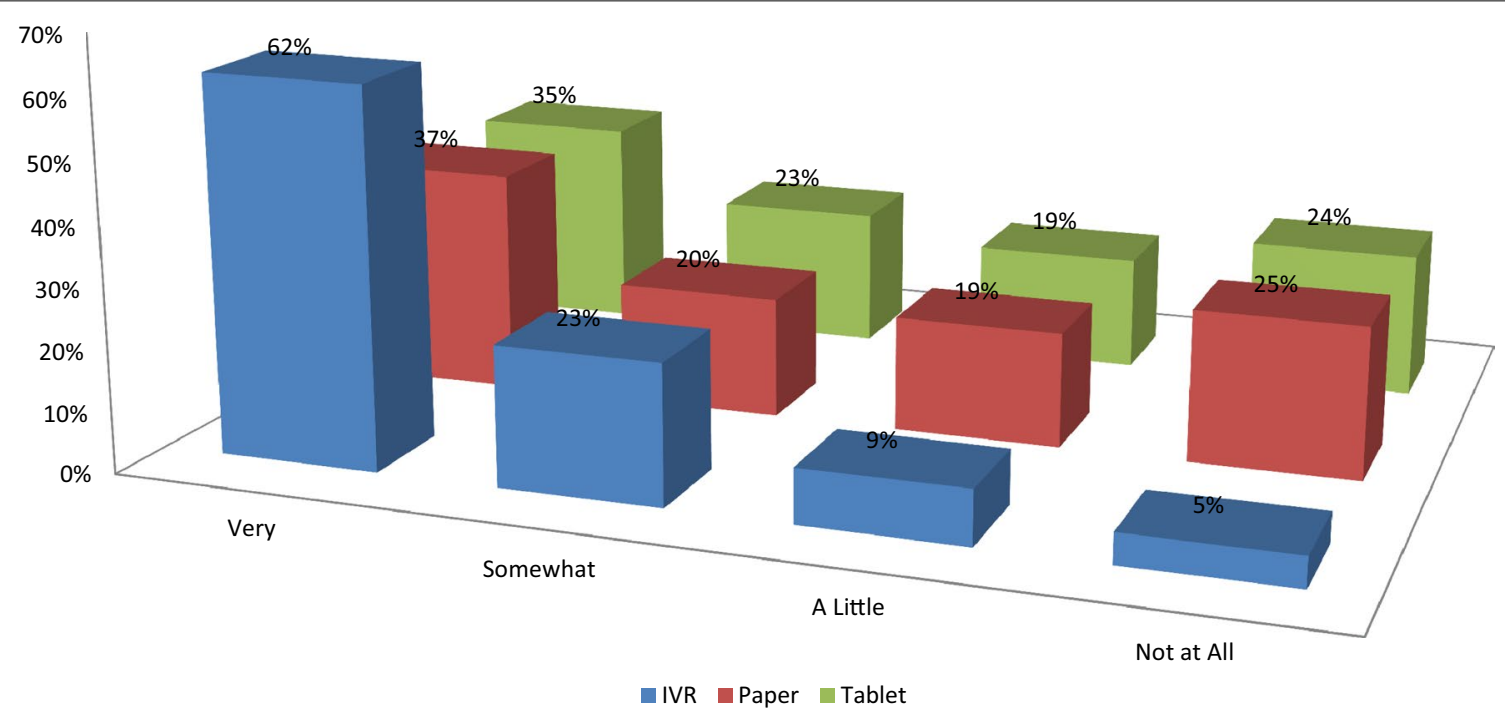

Fig. 6 How willing to use automated telephone survey?

willing to use touchscreen computer or tablet in the future (18-22\% more willing compared to other modes) (Fig. 4). Patients randomized to CSAQ were also 8-9\% more willing to use regular computers with mouse and keyboard than those randomized to other modes (Fig. 5).
Lastly, patients who were randomized to IVR were most likely to respond that they were very willing to use IVR (62\% compared to $37 \%$ for PSAQ and 35\% for CSAQ; Fig. 6). In general, the use of a specific mode positively influenced future receptiveness to that mode. 


\section{Discussion}

The various patient demographic and medical characteristics mostly did not differ by mode. There was no DIF by mode using the lordif methods. Scores for IVR reflected higher function and lower symptoms on average than PSAQ and CSAQ for some domains and measures: When this pattern appeared, it was small effect size (e.g., 1 to 2 score point difference in PROMIS, 0.5 to 0.7 score difference in $0-10 \mathrm{NRS}$ scale, and 0.17 to 0.34 score difference in 1-5 PRO-CTCAE scale). While the confidence intervals for the differences were not completely outside the equivalence margins, the straddle occurred most prominently for IVR compared with other modes in symptom domains. The highest agreement between PSAQ and CSAQ and lowest between PSAQ and IVR were also reported in the systematic review and metaanalysis of studies conducted between 2007 and 2013 [7]. Our randomized parallel groups design removes the potential practice effect, which may explain some of the non-equivalent findings between PSAQ and CSAQ that were not completely within the margin of small effect size.

Patients reporting lower symptoms and higher social or physical functions may suggest that the IVR that used recordings of a pleasant female voice in our study induced social desirability bias, where participants overreported functional well-being and under-reported symptoms as if they were speaking with a real person. If so, this is consistent with the general survey research findings that auditory modes (e.g., IVR/ Phone) yield more positive responses than visual modes [28, 29]. However, social desirability bias for NRS could not be supported in the overall QOL and mental or emotional well-being, because IVR scores were lower on average than CSAQ. The finding that patients were less likely to choose the last option on NRS in these domains could partially explain this anomaly. It is possible that patients were less likely to press two digits to report the highest response option of 10 , (i.e., one and zero), than a single digit on the keypad.

\section{Conclusions}

Because PRO instruments may be administered in a variety of ways, it is critical for the validity of the use of the scores to know if participants would provide the same answers regardless of the modes of administration. Across all comparisons PSAQ-CSAQ, CSAQ-IVR, IVR-PSAQ and across all three kinds of measures, PROMIS, NRS, and PRO-CTCAE, there were some mean differences with CI's upper or lower limit exceeding the margin of small effect. In the current study, the two visual modes (i.e., PSAQ vs CSAQ) agreed more than visual-auditory pairs (i.e., PSAQ vs IVR or CSAQ vs IVR). Several point estimates of score difference lying outside the margin of equivalence suggest that the IVR mode may induce some real differences in scores that are unrelated to the construct being measured, in comparison with PSAQ and CSAQ, depending on the instruments and domains. Primacy effect was supported for IVR in NRS overall QOL and PRO-CTCAE anxiety frequency items. The tendency not to choose the last option was supported for IVR in NRS emotional and mental well-being items, which may be related to participants less willing to record 10 on keypad than single-digit numbers. The next step would to be conduct cognitive interviews to understand these effects for NRS items in IVR. Although the missing data percentages were small in general, there were more missing responses using IVR compared to other modes. The limitation of the study is that we could not differentiate patients who simply did not call in or who broke off the assessment from those whose data have not been saved due to technical problems. Further research should be conducted to understand what contributes to higher missing responses in IVR. In addition, considering some sites experienced issues regarding IVR data storage, the technical aspects of IVR implementation should be checked any time large data collection is planned through this mode. In their meta-analysis, Muehlhausen et al. [7] noted that further research into standards for IVR may be needed to support the equivalence between IVR and other platforms. Because of the non-conclusive equivalence, we may not yet need to consider adjusting for method of data collection when combining data collected via IVR with PSAQ or CSAQ for these PROs. However, because of the greater amount of inconclusive results for IVR, the users of the surveys should consider using IVR only when paper and computer administration is not feasible.

\section{Appendix 1}

See Table 6. 
Table 6 Differential item functioning by modes of administration

\begin{tabular}{|c|c|c|c|}
\hline & $\begin{array}{l}\text { McFadden pseudo } R^{2} \\
\text { change }\left(R_{2}^{2}-R_{1}^{2}\right)\end{array}$ & $\begin{array}{l}\text { McFadden pseudo } R^{2} \\
\text { change }\left(R_{3}^{2}-R_{2}^{2}\right)\end{array}$ & $\begin{array}{l}\text { McFadden pseudo } R^{2} \\
\text { change }\left(R_{3}^{2}-R_{1}^{2}\right)\end{array}$ \\
\hline \multicolumn{4}{|l|}{ Anxiety } \\
\hline I felt fearful & 0.0001 & 0.0007 & 0.0005 \\
\hline I found it hard to focus on anything other than my anxiety & 0.0041 & 0.0051 & 0.0010 \\
\hline My worries overwhelmed me & 0.0019 & 0.0028 & 0.0008 \\
\hline I felt uneasy & 0.0007 & 0.0015 & 0.0008 \\
\hline I felt nervous & 0.0017 & 0.0023 & 0.0006 \\
\hline | felt like I needed help for my anxiety & 0.0015 & 0.0029 & 0.0014 \\
\hline I felt anxious & 0.0002 & 0.0004 & 0.0001 \\
\hline I felt tense & 0.0011 & 0.0015 & 0.0003 \\
\hline (NRS) Describe the level of anxiety on average & 0.0005 & 0.0005 & 0.0000 \\
\hline (PRO-CTCAE) How often did you feel anxiety? & 0.0049 & 0.0058 & 0.0009 \\
\hline (PRO-CTCAE) What was the severity of your anxiety at the WORST? & 0.0025 & 0.0025 & 0.0000 \\
\hline (PRO-CTCAE) How much did anxiety interfere with usual/daily activities? & 0.0009 & 0.0017 & 0.0008 \\
\hline \multicolumn{4}{|l|}{ Depression } \\
\hline I felt that nothing could cheer me up & 0.0144 & 0.0197 & 0.0053 \\
\hline | felt worthless & 0.0000 & 0.0007 & 0.0007 \\
\hline | felt helpless & 0.0011 & 0.0013 & 0.0001 \\
\hline I felt depressed & 0.0055 & 0.0070 & 0.0015 \\
\hline I felt hopeless & 0.0023 & 0.0027 & 0.0004 \\
\hline I felt like a failure & 0.0004 & 0.0009 & 0.0005 \\
\hline I felt unhappy & 0.0041 & 0.0046 & 0.0005 \\
\hline I felt that I had nothing to look forward to & 0.0031 & 0.0033 & 0.0002 \\
\hline (NRS) Describe level of depression on average & 0.0018 & 0.0020 & 0.0002 \\
\hline (PRO-CTCAE) How often did you feel nothing could cheer you up & 0.0014 & 0.0015 & 0.0001 \\
\hline $\begin{array}{l}\text { (PRO-CTCAE) What was the SEVERITY of feelings that nothing could cheer you up at the } \\
\text { WORST? }\end{array}$ & 0.0007 & 0.0007 & 0.0000 \\
\hline (PRO-CTCAE) How much did feeling nothing could cheer you up INTERFERE with activities? & 0.0002 & 0.0008 & 0.0006 \\
\hline (PRO-CTCAE) How OFTEN did you have sad or unhappy feelings & 0.0062 & 0.0066 & 0.0004 \\
\hline (PRO-CTCAE) What was the SEVERITY of your sad/unhappy feelings at the WORST? & 0.0044 & 0.0047 & 0.0003 \\
\hline (PRO-CTCAE) How much did sad/unhappy feelings INTERFERE with activities? & 0.0003 & 0.0003 & 0.0001 \\
\hline \multicolumn{4}{|l|}{ Fatigue } \\
\hline How often did you feel tired? & 0.0005 & 0.0007 & 0.0002 \\
\hline How often did you experience extreme exhaustion? & 0.0002 & 0.0002 & 0.0000 \\
\hline How often did you run out of energy? & 0.0014 & 0.0017 & 0.0003 \\
\hline How often did your fatigue limit you at work? & 0.0001 & 0.0004 & 0.0003 \\
\hline How often were you too tired to think clearly? & 0.0000 & 0.0004 & 0.0004 \\
\hline How often were you too tired to take a bath or shower? & 0.0006 & 0.0012 & 0.0007 \\
\hline How often did you have enough energy to exercise strenuously? & 0.0005 & 0.0013 & 0.0007 \\
\hline How often did you have to push yourself to get things done because of your fatigue? & 0.0023 & 0.0029 & 0.0006 \\
\hline How often did you have trouble finishing things because of your fatigue? & 0.0002 & 0.0005 & 0.0003 \\
\hline (NRS) Describe level of fatigue & 0.0003 & 0.0010 & 0.0007 \\
\hline (PRO-CTCAE) What was severity of fatigue at its worst? & 0.0000 & 0.0002 & 0.0001 \\
\hline (PRO-CTCAE) How much did fatigue interfere with activities? & 0.0000 & 0.0005 & 0.0002 \\
\hline \multicolumn{4}{|l|}{ Pain interference } \\
\hline How much did pain interfere with your family life? & 0.0001 & 0.0002 & 0.0001 \\
\hline How much did pain interfere with work around the home? & 0.0010 & 0.0021 & 0.0011 \\
\hline How much did pain interfere with your ability to participate in social activities? & 0.0026 & 0.0029 & 0.0003 \\
\hline How much did pain interfere with your enjoyment of life? & 0.0002 & 0.0004 & 0.0003 \\
\hline How much did pain interfere with the things you usually do for fun? & 0.0007 & 0.0009 & 0.0002 \\
\hline How much did pain interfere with your enjoyment of social activities? & 0.0002 & 0.0007 & 0.0005 \\
\hline How much did pain interfere with your household chores? & 0.0003 & 0.0009 & 0.0006 \\
\hline (PRO-CTCAE) How much did pain interfere with activities? & 0.0009 & 0.0019 & 0.0010 \\
\hline
\end{tabular}


Table 6 (continued)

\begin{tabular}{|c|c|c|c|}
\hline & $\begin{array}{l}\text { McFadden pseudo } R^{2} \\
\text { change }\left(R_{2}^{2}-R_{1}^{2}\right)\end{array}$ & $\begin{array}{l}\text { McFadden pseudo } R^{2} \\
\text { change }\left(R_{3}^{2}-R_{2}^{2}\right)\end{array}$ & $\begin{array}{l}\text { McFadden pseudo } R^{2} \\
\text { change }\left(R_{3}^{2}-R_{1}^{2}\right)\end{array}$ \\
\hline \multicolumn{4}{|l|}{ Pain intensity } \\
\hline How intense was pain at its worst? & 0.0016 & 0.0026 & 0.0010 \\
\hline How intense was your average pain? & 0.0014 & 0.0018 & 0.0004 \\
\hline What is your level of pain right now? & 0.0001 & 0.0002 & 0.0002 \\
\hline (NRS) Rate severity of pain & 0.0000 & 0.0003 & 0.0003 \\
\hline (PRO-CTCAE) What was severity of pain at its worst? & 0.0005 & 0.0015 & 0.0010 \\
\hline \multicolumn{4}{|l|}{ Physical function } \\
\hline $\begin{array}{l}\text { Does your health now limit you in doing vigorous activities such as running, lifting heavy } \\
\text { objects, participating in strenuous sports? }\end{array}$ & 0.0013 & 0.0018 & 0.0005 \\
\hline Are you able to get on and off the toilet? & 0.0000 & 0.0014 & 0.0014 \\
\hline Does your health now limit you in walking more than a mile? & 0.0010 & 0.0012 & 0.0002 \\
\hline Does your health now limit you in climbing one flight of stairs? & 0.0003 & 0.0003 & 0.0000 \\
\hline Does your health now limit you in lifting or carrying groceries? & 0.0011 & 0.0012 & 0.0001 \\
\hline Does your health now limit you in bending, kneeling, or stooping? & 0.0009 & 0.0015 & 0.0006 \\
\hline Are you able to do chores such as vacuuming or yard work? & 0.0020 & 0.0042 & 0.0022 \\
\hline Are you able to dress yourself, including tying shoelaces and doing buttons? & 0.0018 & 0.0021 & 0.0003 \\
\hline Are you able to shampoo your hair? & 0.0053 & 0.0061 & 0.0008 \\
\hline Are you able to wash and dry your body? & 0.0021 & 0.0043 & 0.0022 \\
\hline (NRS) Overall physical well-being & 0.0003 & 0.0006 & 0.0004 \\
\hline \multicolumn{4}{|l|}{ Sleep disturbance } \\
\hline My sleep quality was & 0.0013 & 0.0023 & 0.0010 \\
\hline My sleep was refreshing & 0.0002 & 0.0005 & 0.0002 \\
\hline I had a problem with my sleep & 0.0007 & 0.0011 & 0.0004 \\
\hline I had difficulty falling asleep & 0.0013 & 0.0021 & 0.0008 \\
\hline My sleep was restless & 0.0007 & 0.0012 & 0.0006 \\
\hline I tried hard to get to sleep & 0.0002 & 0.0006 & 0.0004 \\
\hline I worried about not being able to fall asleep & 0.0003 & 0.0005 & 0.0001 \\
\hline I was satisfied with my sleep & 0.0001 & 0.0001 & 0.0000 \\
\hline (NRS) Describe quality of sleep on average & 0.0002 & 0.0006 & 0.0004 \\
\hline (PRO-CTCAE) What was the severity of your insomnia at its worst? & 0.0039 & 0.0045 & 0.0007 \\
\hline (PRO-CTCAE) How much has insomnia interfered with usual activities? & 0.0045 & 0.0051 & 0.0006 \\
\hline \multicolumn{4}{|l|}{ Ability to participate in social roles and activities } \\
\hline I have trouble doing all of my regular leisure activities with others & 0.0010 & 0.0021 & 0.0011 \\
\hline I have trouble doing all of the family activities that I want to do & 0.0005 & 0.0010 & 0.0005 \\
\hline I have trouble doing all of my usual work (include work at home) & 0.0001 & 0.0009 & 0.0009 \\
\hline I have trouble doing all of the activities with friends that I want to do & 0.0001 & 0.0006 & 0.0004 \\
\hline I have to limit the things I do for fun with others & 0.0002 & 0.0008 & 0.0006 \\
\hline I have to limit my regular activities with friends & 0.0002 & 0.0004 & 0.0001 \\
\hline I have to limit my regular family activities & 0.0008 & 0.0009 & 0.0001 \\
\hline I have trouble doing all of the work that is really important to me (include work at home) & 0.0013 & 0.0016 & 0.0003 \\
\hline (NRS) Rate level of social activity & 0.0007 & 0.0008 & 0.0001 \\
\hline \multicolumn{4}{|l|}{ Global mental health } \\
\hline In general, would you say your quality of life is...? & 0.0008 & 0.0025 & 0.0017 \\
\hline $\begin{array}{l}\text { In general, how would you rate your mental health, including your mood and your ability to } \\
\text { think? }\end{array}$ & 0.0074 & 0.0094 & 0.0019 \\
\hline In general, how would you rate your satisfaction with your social activities and relationships? & 0.0002 & 0.0012 & 0.0010 \\
\hline How would you rate your fatigue on average? & 0.0067 & 0.0069 & 0.0002 \\
\hline (NRS) Rate your overall QOL & 0.0003 & 0.0005 & 0.0002 \\
\hline (NRS) Rate your emotional well-being & 0.0008 & 0.0023 & 0.0015 \\
\hline (NRS) Rate your mental well-being & 0.0035 & 0.0046 & 0.0011 \\
\hline \multicolumn{4}{|l|}{ Global physical health } \\
\hline In general, how would you rate your physical health? & 0.0045 & 0.0049 & 0.0004 \\
\hline
\end{tabular}


Table 6 (continued)

\begin{tabular}{|c|c|c|c|}
\hline & $\begin{array}{l}\text { McFadden pseudo } R^{2} \\
\text { change }\left(R_{2}^{2}-R_{1}^{2}\right)\end{array}$ & $\begin{array}{l}\text { McFadden pseudo } R^{2} \\
\text { change }\left(R_{3}^{2}-R_{2}^{2}\right)\end{array}$ & $\begin{array}{l}\text { McFadden pseudo } R^{2} \\
\text { change }\left(R_{3}^{2}-R_{1}^{2}\right)\end{array}$ \\
\hline $\begin{array}{l}\text { To what extent are you able to carry out everyday physical activities such as walking, climbing } \\
\text { stairs, carrying groceries, or moving a chair? }\end{array}$ & 0.0041 & 0.0043 & 0.0001 \\
\hline Rate fatigue on average & 0.0039 & 0.0041 & 0.0003 \\
\hline (NRS) Rate pain on average & 0.0000 & 0.0001 & 0.0000 \\
\hline (ECOG) Best description of current activity level & 0.0026 & 0.0027 & 0.0001 \\
\hline
\end{tabular}

A base model (model 1) posits that only the trait level predicts responses. A second model (model 2 ) has both trait level and group as independent variables. If model 2 predicts item responses statistically significantly better than model 1 (i.e., McFadden pseudo $R^{2}$ change $\left.\left(R_{2}^{2}-R_{1}^{2}\right) \geq 0.02\right)$, then there is uniform DIF. In uniform DIF, DIF has a consistent impact across trait levels. If the model that includes an interaction term between trait and group (model 3 ) fits significantly better than model 2 (i.e., McFadden pseudo $R^{2}$ change $\left(R_{3}^{2}-R_{2}^{2}\right) \geq 0.02$ ), then the impact of DIF varies by trait level (nonuniform DIF). If model 3 fits significantly better than model 1 (i.e., McFadden pseudo $R^{2}$ change $\left.\left(R_{3}^{2}-R_{1}^{2}\right) \geq 0.02\right)$, there is overall or total DIF

\section{Appendix 2}

See Table 7.

Table 7 Percentages of selecting the first or the last response option in single-item measures

\begin{tabular}{|c|c|c|c|c|c|c|c|}
\hline \multirow[t]{2}{*}{ Group } & \multirow[t]{2}{*}{ PRO } & \multicolumn{3}{|c|}{ Choosing the first option } & \multicolumn{3}{|c|}{ Choosing the last option } \\
\hline & & $\%$ Paper & $\%$ Web & $\%$ IVR & $\%$ Paper & $\%$ Web & $\%$ IVR \\
\hline \multirow[t]{3}{*}{ General health } & NRS overall QOL & 0.0 & 0.2 & 1.4 & 18.4 & 18.3 & 13.7 \\
\hline & NRS emotional well-being & 0.2 & 0.0 & 1.2 & 22.5 & 19.6 & 14.9 \\
\hline & NRS mental well-being & 0.2 & 0.2 & 1.4 & 30.1 & 25.7 & 19.8 \\
\hline Physical function & NRS physical well-being & 0.5 & 0.2 & 0.7 & 13.8 & 11.6 & 14.2 \\
\hline Social function & NRS social activity & 1.4 & 1.2 & 1.2 & 16.4 & 15.2 & 15.7 \\
\hline \multirow[t]{4}{*}{ Anxiety } & NRS anxiety & 25.5 & 28.8 & 30.6 & 0.7 & 0.9 & 0.2 \\
\hline & PRO-CTCAE anxiety frequency & 27.7 & 31.6 & 37.7 & 1.2 & 1.0 & 1.4 \\
\hline & PRO-CTCAE anxiety severity & 31.9 & 34.6 & 39.6 & 1.0 & 0.5 & 1.1 \\
\hline & PRO-CTCAE anxiety interference & 57.7 & 61.0 & 66.4 & 0.7 & 1.5 & 0.4 \\
\hline \multirow[t]{7}{*}{ Depression } & NRS depression & 47.8 & 46.9 & 51.4 & 0.3 & 0.7 & 0.4 \\
\hline & PRO-CTCAE cheer frequency & 57.6 & 59.5 & 63.6 & 1.0 & 1.0 & 0.7 \\
\hline & PRO-CTCAE cheer severity & 63.7 & 66.2 & 68.6 & 0.9 & 0.2 & 0.4 \\
\hline & PRO-CTCAE cheer interference & 67.5 & 72.0 & 73.3 & 0.7 & 0.9 & 0.4 \\
\hline & PRO-CTCAE sad frequency & 27.3 & 30.1 & 29.9 & 0.9 & 1.0 & 0.5 \\
\hline & PRO-CTCAE sad severity & 33.6 & 35.2 & 38.9 & 0.9 & 1.2 & 1.1 \\
\hline & PRO-CTCAE sad interference & 60.9 & 63.6 & 66.8 & 0.9 & 1.0 & 0.4 \\
\hline \multirow[t]{3}{*}{ Fatigue } & NRS fatigue & 10.1 & 11.2 & 12.1 & 1.0 & 1.0 & 0.9 \\
\hline & PRO-CTCAE fatigue severity & 11.3 & 13.0 & 13.2 & 2.9 & 3.1 & 2.7 \\
\hline & PRO-CTCAE fatigue interference & 20.2 & 22.9 & 24.4 & 3.4 & 3.4 & 1.6 \\
\hline \multirow[t]{3}{*}{ Sleep } & NRS sleep overall & 11.2 & 10.4 & 8.1 & 1.2 & 0.5 & 1.8 \\
\hline & PRO-CTCAE sleep severity & 27.3 & 30.8 & 34.9 & 2.7 & 1.4 & 1.8 \\
\hline & PRO-CTCAE sleep interference & 40.5 & 41.3 & 46.9 & 1.5 & 1.9 & 0.9 \\
\hline \multirow[t]{5}{*}{ Pain } & NRS pain frequency & 30.2 & 31.3 & 34.5 & 2.9 & 2.1 & 3.6 \\
\hline & NRS pain severity & 30.0 & 32.6 & 34.7 & 0.7 & 1.2 & 1.1 \\
\hline & PRO-CTCAE pain frequency & 26.1 & 28.1 & 32.0 & 6.3 & 8.2 & 7.5 \\
\hline & PRO-CTCAE pain severity & 28.1 & 30.3 & 34.3 & 2.9 & 2.9 & 3.0 \\
\hline & PRO-CTCAE pain interference & 45.0 & 47.1 & 50.0 & 3.4 & 3.3 & 2.0 \\
\hline
\end{tabular}

The comparisons with statistically significant differences in percentages after Bonferroni correction are bolded 


\section{Appendix 3}

See Table 8.

Table 8 Missing data percentage by items and modes in PROMIS items

\begin{tabular}{|c|c|c|c|c|c|c|c|c|c|c|}
\hline PROMIS short forms & V1 (\%) & V2 (\%) & V3 (\%) & V4 (\%) & V5 (\%) & V6 (\%) & V7 (\%) & V8 (\%) & V9 (\%) & V10 (\%) \\
\hline \multicolumn{11}{|l|}{ Pain intensity } \\
\hline PSAQ & 1 & 1 & 2 & & & & & & & \\
\hline IVR & 6 & 6 & 6 & & & & & & & \\
\hline CSAQ & 1 & 1 & 1 & & & & & & & \\
\hline \multicolumn{11}{|l|}{ Anxiety } \\
\hline PSAQ & 2 & 2 & 2 & 2 & 2 & 2 & 3 & 2 & 2 & \\
\hline IVR & 5 & 5 & 6 & 5 & 6 & 5 & 6 & 5 & 5 & \\
\hline CSAQ & 1 & 1 & 1 & 1 & 1 & 2 & 1 & 2 & 2 & \\
\hline \multicolumn{11}{|l|}{ Depression } \\
\hline PSAQ & 3 & 3 & 3 & 3 & 3 & 3 & 3 & 3 & & \\
\hline IVR & 5 & 5 & 5 & 5 & 5 & 5 & 5 & 5 & & \\
\hline CSAQ & 1 & 2 & 2 & 2 & 1 & 2 & 2 & 2 & & \\
\hline \multicolumn{11}{|l|}{ Fatigue } \\
\hline PSAQ & 2 & 2 & 2 & 3 & 2 & 2 & 3 & 2 & 2 & \\
\hline IVR & 6 & 6 & 6 & 6 & 7 & 6 & 6 & 6 & 6 & \\
\hline CSAQ & 1 & 1 & 1 & 1 & 1 & 1 & 1 & 1 & 1 & \\
\hline \multicolumn{11}{|l|}{ Pain interference } \\
\hline PSAQ & 1 & 1 & 1 & 1 & 1 & 1 & 1 & & & \\
\hline IVR & 6 & 6 & 6 & 6 & 6 & 6 & 6 & & & \\
\hline CSAQ & 1 & 1 & 1 & 1 & 1 & 1 & 1 & & & \\
\hline \multicolumn{11}{|l|}{ Sleep disturbance } \\
\hline PSAQ & 2 & 1 & 1 & 1 & 1 & 2 & 2 & 1 & & \\
\hline IVR & 6 & 6 & 6 & 6 & 7 & 6 & 6 & 6 & & \\
\hline CSAQ & 1 & 1 & 1 & 1 & 1 & 1 & 1 & 1 & & \\
\hline \multicolumn{11}{|l|}{ Social roles and activities } \\
\hline PSAQ & 2 & 2 & 2 & 2 & 2 & 2 & 2 & 2 & 3 & \\
\hline IVR & 5 & 5 & 5 & 5 & 5 & 5 & 6 & 5 & 5 & \\
\hline CSAQ & 1 & 1 & 1 & 1 & 1 & 1 & 2 & 2 & 3 & \\
\hline \multicolumn{11}{|l|}{ Physical function } \\
\hline PSAQ & 2 & 1 & 1 & 2 & 2 & 2 & 2 & 3 & 1 & 1 \\
\hline IVR & 4 & 4 & 4 & 4 & 4 & 4 & 4 & 4 & 4 & 4 \\
\hline CSAQ & 1 & 1 & 1 & 1 & 1 & 1 & 1 & 1 & 1 & 1 \\
\hline
\end{tabular}

The columns from V1 through V10 indicate simply items administered by order in each scale

\section{Abbreviations}

PROMIS: Patient-Reported Outcomes Measurement System; PRO-CTCAE: Patient-Reported Outcomes version of the Common Terminology Criteria for Adverse Events; NRS: Numerical rating scale; IVR: Interactive voice response; DIF: Differential item functioning; PRO: Patient-reported outcomes; PSAQ: Paper and pencil self-administered questionnaire; CSAQ: Computerized selfadministered questionnaire.

Acknowledgements

Not applicable.

\section{Authors' contributions}

Conceptualization: All authors; Data analyses: Novotny, Lee; Original draft preparation: Lee, Beebe, Novotny; Review and editing: all authors; Funding acquisition and supervision: Sloan. All authors read and approved the final manuscript.

\section{Funding}

This study was supported by National Cancer Institute Grants R01CA154537 (Sloan) and P30CA015083 (Diasio). Lee was additionally supported by the Robert D. and Patricia E. Kern Center for the Science of Health Care Delivery at Mayo Clinic.

Availability of data and materials

Data can be made available upon reasonable request to the principal investigator (J. Sloan). All requests will be reviewed. 


\section{Declarations}

\section{Ethics approval and consent to participate}

The study was reviewed by the IRB of each of the participating sites, and all patients provided consent to enter the study.

\section{Consent for publication}

Not applicable.

\section{Competing interests}

The authors declare that they have no competing interest to report.

\section{Author details}

'Department of Quantitative Health Sciences, Mayo Clinic, 200 First St SW, Rochester, MN 55905, USA. ${ }^{2}$ Division of Health Policy and Management, University of Minnesota School of Public Health, 625 Michigan Ave, 27th Floor, Chicago, IL 60611, USA. ${ }^{3}$ Department of Quantitative Health Sciences, Mayo Clinic, 13400 E. Shea Blvd., Scottsdale, AZ 85259, USA.

Received: 6 February 2021 Accepted: 4 September 2021

Published online: 17 September 2021

\section{References}

1. Smith SK, Rowe K, Abernethy AP (2014) Use of an electronic patientreported outcome measurement system to improve distress management in oncology. Palliat Support Care 12(1):69-73

2. Kroenke K et al (2021) Choosing and using patient-reported outcome measures in clinical practice. Arch Phys Med Rehabil

3. Porter I et al (2016) Framework and guidance for implementing patientreported outcomes in clinical practice: evidence, challenges and opportunities. J Comp Eff Res 5(5):507-519

4. Broering JM et al (2014) Measurement equivalence using a mixed-mode approach to administer health-related quality of life instruments. Qual Life Res 23(2):495-508

5. Campbell $\mathrm{N}$ et al (2015) Equivalence of electronic and paper-based patient-reported outcome measures. Qual Life Res 24(8):1949-1961

6. Gwaltney CJ, Shields AL, Shiffman S (2008) Equivalence of electronic and paper-and-pencil administration of patient-reported outcome measures: a meta-analytic review. Value Health 11(2):322-333

7. Muehlhausen $W$ et al (2015) Equivalence of electronic and paper administration of patient-reported outcome measures: a systematic review and meta-analysis of studies conducted between 2007 and 2013. Health Qual Life Outcomes 13:167

8. Mulhern B et al (2015) Comparing the measurement equivalence of EQ-5D-5L across different modes of administration. Health Qual Life Outcomes 13:191

9. Rasmussen SL et al (2016) High level of agreement between electronic and paper mode of administration of a thyroid-specific patient-reported outcome, ThyPRO. Eur Thyroid J 5(1):65-72

10. Rutherford C et al (2016) Mode of administration does not cause bias in patient-reported outcome results: a meta-analysis. Qual Life Res 25(3):559-574

11. Bennett AV et al (2016) Mode equivalence and acceptability of tablet computer-, interactive voice response system-, and paper-based administration of the U.S. National Cancer Institute's Patient-Reported Outcomes version of the Common Terminology Criteria for Adverse Events (PRO CTCAE). Health Qual Life Outcomes 14:24
12. Bjorner JB et al (2014) Difference in method of administration did not significantly impact item response: an IRT-based analysis from the Patient-Reported Outcomes Measurement Information System (PROMIS) initiative. Qual Life Res 23(1):217-227

13. Lundy JJ et al (2020) Agreement among paper and electronic modes of the EQ-5D-5L. Patient Patient Cent Outcomes Res 13(4):435-443

14. Abu-Hasaballah K, James A, Aseltine RH Jr (2007) Lessons and pitfalls of interactive voice response in medical research. Contemp Clin Trials 28(5):593-602

15. Weiler K et al (2004) Quality of patient-reported outcome data captured using paper and interactive voice response diaries in an allergic rhinitis study: is electronic data capture really better? Ann Allergy Asthma Immunol 92(3):335-339

16. Shea JA et al (2008) Adapting a patient satisfaction instrument for low literate and Spanish-speaking populations: comparison of three formats. Patient Educ Couns 73(1):132-140

17. Singh JA et al (2014) Normative data and clinically significant effect sizes for single-item numerical linear analogue self-assessment (LASA) scales. Health Qual Life Outcomes 12:187

18. Chew LD et al (2008) Validation of screening questions for limited health literacy in a large VA outpatient population. J Gen Intern Med 23(5):561-566

19. Edwards MC, Houts CR, Wirth RJ (2018) Measurement invariance, the lack thereof, and modeling change. Qual Life Res 27(7):1735-1743

20. Meredith W, Teresi JA (2006) An essay on measurement and factorial invariance. Med Care 44:569-77

21. Choi SW, Gibbons LE, Crane PK (2011) Iordif: an R package for detecting differential item functioning using iterative hybrid ordinal logistic regression/item response theory and Monte Carlo simulations. J Stat Softw 39(8):1-30

22. Rosseel Y (2012) lavaan: an R package for structural equation modeling. J Stat Softw 48(2):1-36

23. Mueller RO, Hancock GR (2008) Best practices in structural equation modeling. In: Osborne J (ed) Best practices in quantitative methods. Sage Publications, Thousand Oaks, pp 488-508

24. McDonald RP (1999) Test theory: a unified treatment. Erlbaum, Mahwah

25. Reise SP, Scheines R, Widaman KF, Haviland MG (2013) Multidimensionality and structural coefficient bias in structural equation modeling: a bifactor perspective. Educ Psychol Measur 73:5-26

26. Condon DM et al (2020) Does recall period matter? Comparing PROMIS((R)) physical function with no recall, 24-hr recall, and 7-day recall. Qual Life Res 29(3):745-753

27. Coons SJ et al (2009) Recommendations on evidence needed to support measurement equivalence between electronic and paper-based patientreported outcome (PRO) measures: ISPOR ePRO Good Research Practices Task Force report. Value Health 12(4):419-429

28. Elliott MN et al (2013) A randomized experiment investigating the suitability of speech-enabled IVR and Web modes for publicly reported surveys of patients' experience of hospital care. Med Care Res Rev 70(2):165-184

29. French KA, Falcon CN, Allen TD (2019) Experience sampling response modes: comparing voice and online surveys. J Bus Psychol 34:575-586

\section{Publisher's Note}

Springer Nature remains neutral with regard to jurisdictional claims in published maps and institutional affiliations. 